\title{
CDK12 is a transcription elongation- associated CTD kinase, the metazoan ortholog of yeast Ctk1
}

\author{
Bartlomiej Bartkowiak, ${ }^{1,5}$ Pengda Liu, ${ }^{1,5}$ Hemali P. Phatnani, ${ }^{1,6}$ Nicholas J. Fuda, $^{2}$ Jeffrey J. Cooper, $^{3}$ \\ David H. Price, ${ }^{3}$ Karen Adelman, ${ }^{4}$ John T. Lis, ${ }^{2}$ and Arno L. Greenleaf ${ }^{1,7}$ \\ ${ }^{1}$ Department of Biochemistry, Duke Center for RNA Biology, Duke University Medical Center, Durham, North Carolina 27710, \\ USA; ${ }^{2}$ Department of Molecular Biology and Genetics, Cornell University, Ithaca, New York 14850, USA; ${ }^{3}$ Department of \\ Biochemistry, University of Iowa, Iowa City, Iowa 52242, USA; ${ }^{4}$ Laboratory of Molecular Carcinogenesis, National Institute of \\ Environmental Health Sciences, Research Triangle Park, North Carolina 27709, USA
}

\begin{abstract}
Drosophila contains one (dCDK12) and humans contain two (hCDK12 and hCDK13) proteins that are the closest evolutionary relatives of yeast $\mathrm{Ctk} 1$, the catalytic subunit of the major elongation-phase $\mathrm{C}$-terminal repeat domain (CTD) kinase in Saccharomyces cerevisiae, CTDK-I. However, until now, neither CDK12 nor CDK13 has been demonstrated to be a bona fide CTD kinase. Using Drosophila, we demonstrate that dCDK12 (CG7597) is a transcription-associated CTD kinase, the ortholog of yCtk1. Fluorescence microscopy reveals that the distribution of dCDK12 on formaldehyde-fixed polytene chromosomes is virtually identical to that of hyperphosphorylated RNA polymerase II (RNAPII), but is distinct from that of P-TEFb (dCDK9 + dCyclin T). Chromatin immunoprecipitation (ChIP) experiments confirm that dCDK12 is present on the transcribed regions of active Drosophila genes. Compared with P-TEFb, dCDK12 amounts are lower at the $5^{\prime}$ end and higher in the middle and at the 3' end of genes (both normalized to RNAPII). Appropriately, Drosophila dCDK12 purified from nuclear extracts manifests CTD kinase activity in vitro. Intriguingly, we find that cyclin $\mathrm{K}$ is associated with purified dCDK12, implicating it as the cyclin subunit of this CTD kinase. Most importantly, we demonstrate that RNAi knockdown of dCDK12 in S2 cells alters the phosphorylation state of the CTD, reducing its Ser2 phosphorylation levels. Similarly, in human HeLa cells, we show that hCDK13 purified from nuclear extracts displays CTD kinase activity in vitro, as anticipated. Also, we find that chimeric (yeast/human) versions of Ctk1 containing the kinase homology domains of hCDK12/13 (or hCDK9) are functional in yeast cells (and also in vitro); using this system, we show that a bur1 ${ }^{t s}$ mutant is rescued more efficiently by a hCDK9 chimera than by a hCDK13 chimera, suggesting the following orthology relationships: Bur1 $\leftrightarrow$ CDK9 and Ctk1 $\leftrightarrow$ CDK12/13. Finally, we show that siRNA knockdown of hCDK12 in HeLa cells results in alterations in the CTD phosphorylation state. Our findings demonstrate that metazoan CDK12 and CDK13 are CTD kinases, and that CDK12 is orthologous to yeast Ctk1.
\end{abstract}

[Keywords: CTD kinase; transcription; RNA polymerase II; C-terminal repeat domain; P-TEFb]

Supplemental material is available at http://www.genesdev.org.

Received July 9, 2010; revised version accepted August 26, 2010.

The C-terminal repeat domain (CTD) of RNA polymerase II (RNAPII) is an intrinsically unstructured extension of the enzyme's largest subunit, RPB1. The CTD is composed of a tandem array of seven amino acid repeats with the consensus sequence $\mathrm{Y}_{1} \mathrm{~S}_{2} \mathrm{P}_{3} \mathrm{~T}_{4} \mathrm{~S}_{5} \mathrm{P}_{6} \mathrm{~S}_{7}$; the number of heptad repeats varies from organism to organism and appears to correlate with genomic complexity (Corden

\footnotetext{
${ }^{5}$ These authors contributed equally to this work.

${ }^{6}$ Present address: Department of Biochemistry and Molecular Biophysics, Columbia University Medical Center, New York, NY 10032, USA. ${ }^{7}$ Corresponding author.

E-MAIL arno@duke.edu; FAX (919) 684-8885.

Article is online at http://www.genesdev.org/cgi/doi/10.1101/gad.1968210.
}

1990). Although dispensable for polymerase activity in vitro, the CTD is conserved throughout evolution and is essential to life (Zehring et al. 1988). The CTD's function is to coordinate transcription with nuclear processes such as mRNA processing and chromatin modification; it fulfills this role by serving as a selective binding scaffold for nuclear factors (for review, see Phatnani and Greenleaf 2006). The binding specificity of the CTD for particular factors is determined by its phosphorylation state, which varies during the transcription cycle; Ser2, Ser5, and, more recently, Ser7 of the heptad repeat have been identified as the primary targets of phosphorylation (Phatnani and Greenleaf 2006; Chapman et al. 2007; 
Egloff et al. 2007). Initiated and early transcribing polymerases are thought to be phosphorylated at Ser5 positions in the CTD repeats, whereas actively elongating polymerases carry phosphates on both Ser2 and Ser5 residues. Toward the $3^{\prime}$ end of pre-mRNA transcription units, Ser2 phosphorylation often appears to predominate (cf. Phatnani and Greenleaf 2006; Rahl et al. 2010).

CTD kinases in yeast and metazoa The phosphorylation of CTD Ser5 residues during initiation and early transcription is mediated by the TFIIH CTD kinase subunit: Kin28 in yeast, and CDK7 in metazoans (Komarnitsky et al. 2000; Schroeder et al. 2000). The majority of Ser2 phosphorylation on productively elongating RNAPII in Saccharomyces cerevisiae $(S c)$ appears to be catalyzed by CTDK-I (Buratowski 2009; Qiu et al. 2009), a threesubunit enzyme consisting of Ctk1 (a CDK homolog), Ctk2 (a cyclin homolog), and Ctk3 (function unknown) (Lee and Greenleaf 1991; Sterner et al. 1995). Although it is responsible for the bulk of Ser2 phosphorylation in vivo, the Ctk1-containing enzyme is not the only elongation phase kinase in yeast; it coexists with the essential Bur1 kinase $(\mathrm{CDK} /$ cyclin pair = Bur1/Bur2) (Yao et al. 2000). While it has been proposed that the Burl kinase phosphorylates the transcription elongation factor subunit SPT5 rather than the CTD of Rpb1 (Zhou et al. 2009), recent evidence suggests that the situation is more complex, and that, in addition to acting on SPT5, the Bur1 kinase phosphorylates Ser2 positions in the CTD (Liu et al. 2009; Qiu et al. 2009), possibly stimulating subsequent phosphorylation by Ctk1. A similar pair of Ser2 CTD kinases is also present in the fission yeast Schizzosaccharomyces pombe $(S p)$ : Lsk1, the Ctk1 ortholog in $S p$, phosphorylates Ser2 positions in the CTD (Karagiannis and Balasubramanian 2007; Coudreuse et al. 2010), whereas SpCDK9, the Bur1 ortholog, phosphorylates both Rpb1 (on its CTD) and Spt5 (Viladevall et al. 2009).

With respect to the principal Ser2 kinase in metazoa, the situation has been less clear cut. It is currently thought that there is only one Ser2 CTD kinase in higher organisms: P-TEFb (consisting of CDK9 and a cyclin, usually cyclin T) (e.g., see Peterlin and Price 2006). P-TEFb is able to phosphorylate both the CTD and the elongation factor subunit SPT5 (Kim and Sharp 2001). The dual functionality of CDK9, coupled with its equal sequence similarity to both Burl and Ctk1, has led to the assumption that CDK9 reconstitutes the activities of both Bur1 and Ctk1 in higher eukaryotes (Wood and Shilatifard 2006). On the other hand, two independent molecular evolution studies concluded that Sc Burl is the closest Sc relative of metazoan CDK9 proteins, while $S c$ Ctk1 is closer to a set of metazoan CDK proteins that are largely unstudied (Liu and Kipreos 2000; Guo and Stiller 2004): the "Ctk1" group (Supplemental Fig. S1). The genomes of Drosophila melanogaster $(D m)$ and Caenorhabditis elegans $(C e)$ each encode one member of the Ctk1 group (genes CG7597 and B0285.1, respectively), while the genome of Homo sapiens (Hs) encodes two (genes CRKRS and CDC2L5; see Supplemental Fig S1). The human "Ctk1" proteins have been renamed recently CDK12 and CDK13 (Chen et al. 2006,
2007). We refer to them as hCDK12 and hCDK13, and to the $D m$ protein as dCDK12.

The evolutionary studies thus suggest that the metazoan ortholog of Ctk1 in Drosophila is dCDK12 and in humans is one or both of the isozyme pair, hCDK12+ hCDK13. Also, the evolutionary arguments and the recent experimental work mentioned above both suggest that the metazoan ortholog of Bur1 is CDK9. We tested these suggestions with a set of diverse experiments that lead us to conclude that, indeed, the Drosophila ortholog of yeast Ctk1 is dCDK12 (CG7597), and the human ortholog is most likely hCDK12. Conversely, we describe other experiments supporting the idea that the metazoan ortholog of yeast Bur1 is CDK9. Because the metazoan orthologs of yeast Ctk1 have not been studied previously as CTD kinases, our findings open the door to novel studies of a vast array of transcription-related events in metazoa that are critical to many aspects of proper gene expression and other nuclear functions.

\section{Results}

Drosophila CDK12 localizes to active genes in vivo

dCDK12 kinase colocalizes with RNAP IIO on polytene chromosomes Given the evolutionary relatedness of CDK12/13 and Ctk1, we sought to compare their functional characteristics. Because Ctk1 is found across transcription units in parallel with hyperphosphorylated RNAPII (Cho et al. 2001; Qiu et al. 2009), we tested whether such colocalization also occurred for dCDK12 and hyperphosphorylated RNAPII (Pol IIO) on Drosophila polytene chromosomes. Affinity-purified rabbit antidCDK12 IgGs (see Supplemental Fig. S2) were used in concert with affinity-purified goat anti-IIO IgGs (Morris et al. 1997) to reveal the distributions of dCDK12 and hyperphosphorylated RNAPII on formaldehyde-fixed polytene chromosomes (Greenleaf et al. 1976; Weeks et al. 1993; Schwartz et al. 2004). As shown in Figure 1, $\mathrm{A}$ and $\mathrm{B}$, the distributions of Pol IIO and CDK12 are virtually identical, both on chromosomes from animals grown under normal conditions and from animals subjected to heat shock. Clearly discernable loci that stain for Pol IIO also stain for CDK12, with basically the same pattern of staining (in the merged images in Fig. 1A,B, there are essentially no only red or only green areas of staining). The codistribution of CDK12 and PolIIO is further illustrated in Figure 1C for one chromosome segment (non-heat shock) shown in a split view: half stained for CDK12, and half stained for Pol IIO. Another segment (heat-shocked) in split-view close-up is shown in Figure 1D. These images illustrate that virtually all actively elongating RNAPII (Pol IIO) is accompanied by CDK12. This finding supports the idea that CDK12 kinase is important to RNAPII function during the elongation phase of transcription, most likely through phosphorylating the CTD of Rpb1.

Chromosomal distributions of $d C D K 12$ and P-TEFb $(C D K 9+$ Cyclin T) In the absence of highly specific 
A

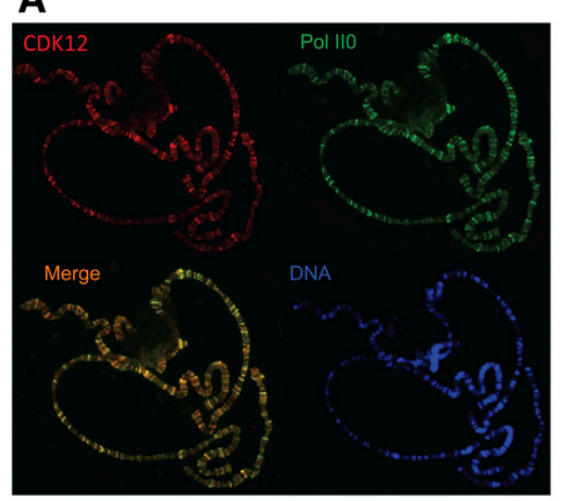

C

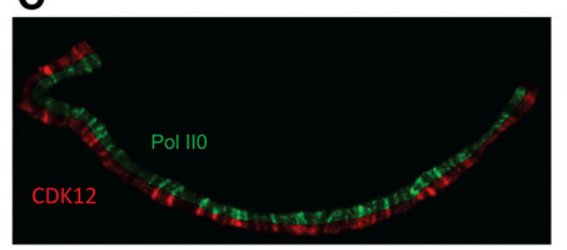

B

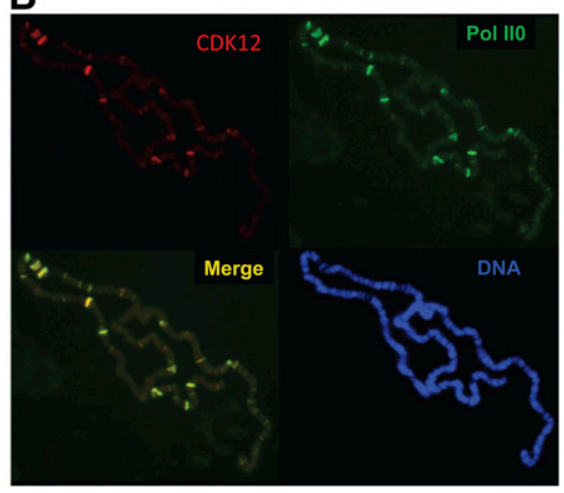

D

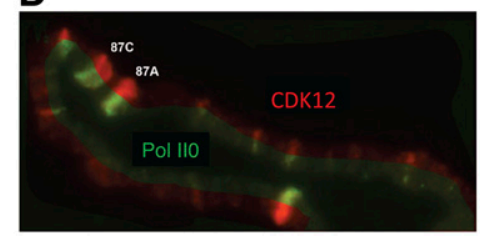

Figure 1. Distribution of $\mathrm{dCDK} 12$ and hyperphosphorylated RNAPII (Pol IIO) on $D m$ polytene chromosomes, determined by immunofluorescence. $(A)$ Chromosomes from NHS larva reacted with anti-dCDK12 (red), anti-IIO (green), or DAPI (blue: "DNA"). Merge is an overlay of the red and green images. (B) Chromosome set from a heatshocked larva $\left(20 \mathrm{~min}\right.$ at $\left.37^{\circ} \mathrm{C}\right) .(\mathrm{C})$ Isolated segment of a chromosome arm (non-heat shock) showing split images of dCDK12 (red) and Pol II0 (green). (D) Segment of chromosome 3R showing split images of dCDK12 and Pol IIO. Major heat-shock puffs are labeled (87A and $87 \mathrm{C}$ ). antibodies directed against dCDK9, we used an affinitypurified antibody to dCyclin $\mathrm{T}$ to compare directly the distribution of CDK12 kinase with that of P-TEFb (Lis et al. 2000) on polytene chromosomes. The images in Figure 2 demonstrate that the distributions of the two kinases show overlap, but they also display some striking quantitative and qualitative differences. On non-heatshocked (NHS) chromosomes, ecdysone-induced developmental puffs stain intensely for CDK12 (as they do for
RNAP II0), but weakly for Cyclin T (e.g., Fig. 2A, white arrow). There are also sites with a strong signal for Cyclin $\mathrm{T}$ and little if any signal for CDK12 (e.g., Fig. 2A, green dots in merge panel); conversely, a number of sites that are very bright for CDK12 are negative or nearly negative for Cyclin T (Fig. 2A, red dots).

In general, transcription units at developmentally regulated loci are different from heat-shock genes in that they are much longer and contain introns. A chromosome
A
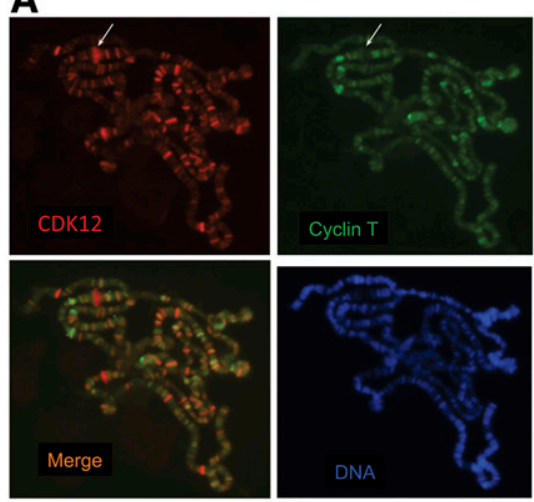

C

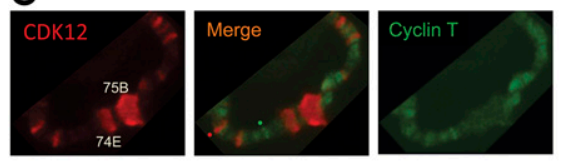

B

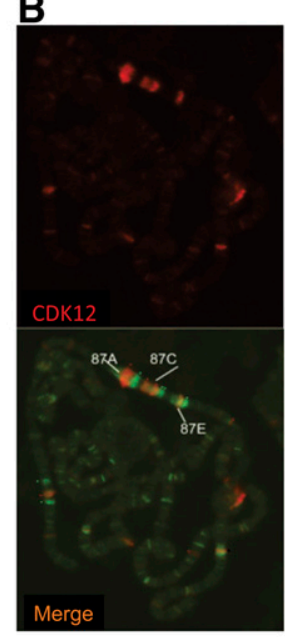

D

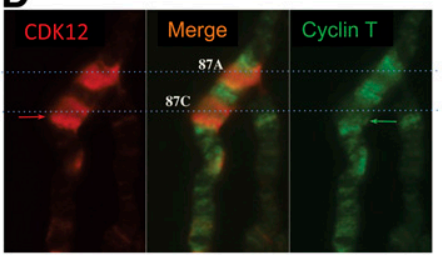

Figure 2. Distribution of $\mathrm{dCDK} 12$ and dCyclin $\mathrm{T}$ on polytene chromosomes. $(A)$ Chromosomes from NHS larva reacted with anti-dCDK12 (red), anti-dCyclin T (green), or DAPI (blue: "DNA"). The white arrow indicates a developmental puff that stains strongly for CDK12, but weakly for Cyclin T. Red and green dots in the Merge panel indicate bands that are predominantly red (CDK12) or green (Cyclin T), respectively. (B) Chromosome set from a heat-shocked larva $\left(20 \mathrm{~min}\right.$ at $\left.37^{\circ} \mathrm{C}\right)$ analyzed as for $A$. Red and green dots in the Merge panel indicate bands that are almost exclusively red (CDK12) or green (Cyclin T), respectively. $(C)$ Isolated chromosome segment (non-heat shock) showing CDK12 (red) and Cyclin T (green). Prominent developmental (ecdysone-induced) puffs $74 \mathrm{E}$ and $75 \mathrm{~B}$ are labeled. Colored dots indicate sites that stain almost uniquely for the corresponding protein. $(D)$ Segment of chromosome 3R (heat shock) with the merged image aligned between images of CDK12 and Cyclin T. Major heat-shock loci are labeled (87A and $87 \mathrm{C}$ ). Dotted lines facilitate comparisons between red and green images. 
segment (NHS) containing developmental puffs at 74E and $75 \mathrm{~B}$ is shown enlarged in Figure 2C. These two puffs stain strongly for dCDK12 but weakly for dCyclin T. Other sites with high relative labeling intensity for CDK12 or Cyclin $\mathrm{T}$ are indicated in Figure $2 \mathrm{C}$ by a red or green dot, respectively.

Heat-shock-induced puffs show very different staining patterns for CDK12 versus Cyclin T (Fig. 2B), in contrast to CDK12 versus Pol II0, which are very similarly distributed on heat-shock puffs (Fig. 1D). For example, at $87 \mathrm{C}$, two bright bands of CDK12 (Fig. 2B, red) are often observed "flanking" a middle band of Cyclin T (Fig. 2B, green, merge). Closer inspection reveals that Cyclin T staining also occurs in a punctate pattern "under" the more diffuse CDK12 pattern (Fig. 2B,D, cf. red and green). In a close-up view of heat-shock puffs $87 \mathrm{~A}$ and $87 \mathrm{C}$ (Fig. 2D), it can be seen that the intensities for the two proteins differ "along" a puff. For example, below the bottom dotted line in Figure 2D there is a band that stains both green (right) and red (left); in the merged image ("both"), it appears yellowish. However, moving up from that band (along the chromosome axis) the green intensity drops and remains low, whereas the red intensity drops and then increases again (Fig. 2D). Thus, dCDK12 and P-TEFb associate with chromosomes in partially overlapping but distinct distributions. Our results therefore support the idea that dCDK9 and dCDK12 perform distinct functions at specific locations along the transcription unit.

Distributions of dCDK12 across individual genes To determine the distribution of dCDK12 at a higher spatial resolution, we used chromatin immunoprecipitation (ChIP) and Drosophila cultured cells, as used previously for dP-TEFb (Boehm et al. 2003). On the well-studied Hsp70 gene, anti-Rpb3 antibody detected the typical presence of promoter-proximally paused polymerase at the 5 ' end prior to heat shock (Fig. 3B, "NHS" at -154 and +96); after a 10-min heat shock (Fig. 3B, 10'HS), RNAPII presence increased dramatically along the entire transcription unit, with a peak at +96 still present. Analyzing for dCDK12 revealed no signals above background before heat shock, but significant signals along virtually the entire gene after heat shock (Fig. 3A). The results thus indicate no presence of dCDK12 with the paused RNAPII before gene activation, and they show that the promoterproximal peak of RNAPII at +96 after activation is not accompanied by a similar peak of dCDK12. The ratio of dCDK12 to RNAPII shows a rise to maximum level at the +379 position, and the amount of dCDK12 relative to polymerase remains about constant throughout the gene (Fig. 3C). These results contrast with those for P-TEFb (Boehm et al. 2003), which showed a concentration that peaked at the $5^{\prime}$ end of the gene (+58 in that study) and steadily declined along the gene. When the ratio of $\mathrm{P}$-TEFb to RNAPII was plotted, values in the $3^{\prime}$ half of the gene were lower than in the $5^{\prime}$ half (Fig. 4D in Boehm et al. 2003). The ChIP results, therefore, are consistent with our polytene chromosome-staining results in showing different distributions for $\mathrm{dCDK} 12$ and $\mathrm{dP}-\mathrm{TEFb}$ on the Hsp70 gene.
Because the polytene localization experiments also indicated differences between distributions of dCDK12 and $\mathrm{dP}-\mathrm{TEFb}$ on non-heat-shock genes (Fig. 2A,C), we analyzed four such loci by ChIP. These four genes displayed RNAPII amounts that were highest at the $5^{\prime}$ end and decreased toward the 3 ' end (Fig. 3E), although the relative height of the $5^{\prime}$ peak differed from gene to gene. In contrast, dCDK12 amounts were more constant across the genes (Fig. 3D), and two of the four genes displayed higher signals at internal positions than at the 5'-most position (exba and Rp49). The ratio of dCDK12 signal to RNAPII is plotted for the non-heat-shock genes in Figure 3F, and for all genes is seen to be higher at internal and $3^{\prime}$ positions than at 5 ' end-proximal positions. These results again distinguish $\mathrm{dCDK} 12$ from $\mathrm{dP}$ $\mathrm{TEFb}$ in spatial distribution, and suggest that the two kinases have different functions.

\section{Drosophila CDK12 and human CDK13 manifest CTD kinase activity in vitro}

dCDK12 exhibits CTD kinase activity We purified dCDK12 from nuclear extract (KcN) of Drosophila cultured cells (line Kc) (Price et al. 1987) using polyacrylamide beads carrying affinity-purified anti-dCDK12 IgGs (see above; Materials and Methods). After extensive washing at up to $0.4 \mathrm{M} \mathrm{NaCl}$, the activity on the beads was eluted with antigenic peptide into three fractions (E1, E2, and E3), and the eluted fractions were assayed for kinase activity on a $\beta$-galactosidase-Drosophila CTD fusion protein ( $\beta$ galdCTD) (Fig. 4A). CTD kinase activity was observed in fractions E1, E2, and E3 (order of decreasing activity), while no kinase activity was observed in the absence of added enzyme (Fig. 4A, "no E" control lane). In this experiment, radioactivity was incorporated into both an unshifted band at the $\beta$ gal-dCTD mobility (IIa analog) and a shifted band with reduced mobility (IIo analog). In a second pull-down experiment, the antibody beads were washed in even higher salt $(0.8 \mathrm{M} \mathrm{NaCl})$ and then assayed for bead-bound kinase activity-in this case, on a GST-yCTD fusion protein (Fig. 4B). The antibody beads displayed kinase activity on the GST-yCTD substrate (Fig. 4B, Beads + CTD lane), while the controls showed no activity (Fig. 4B, CTD alone and Beads alone). In this case, the main product band was a mobility-shifted form of the fusion protein migrating slower than the unmodified GST-yCTD. While the kinase is present in amounts too small to be seen in the stained gel here, we know it has been purified extensively (Fig. 5; see below). Together, these results demonstrate that CDK12 from Drosophila cells exhibits CTD kinase activity.

hCDK13 exhibits CTD kinase activity To determine whether human CDK13 also exhibits CTD kinase activity, we purified it by a combination of standard methods and immunoaffinity-based approaches. The hCDK 13 present in a nuclear extract from HeLa cells bound to MacroPrep CM resin (Bio-Rad) and was eluted by $0.6 \mathrm{M} \mathrm{NaCl}$ (Materials and Methods). We used immunoaffinity beads carrying anti-hCDK13 IgGs to further purify the kinase. 
A.

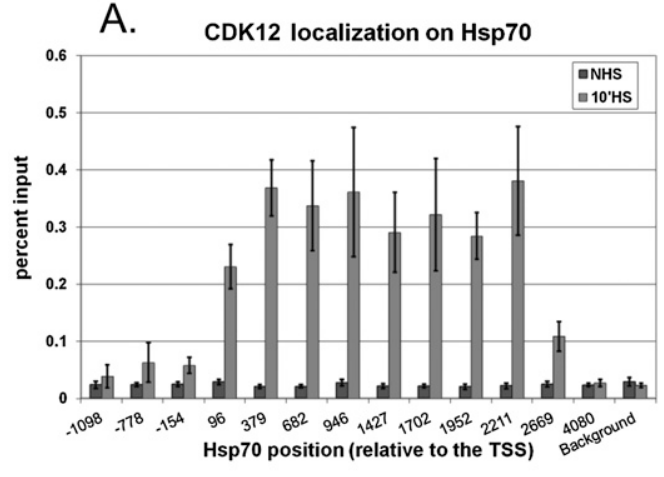

B.

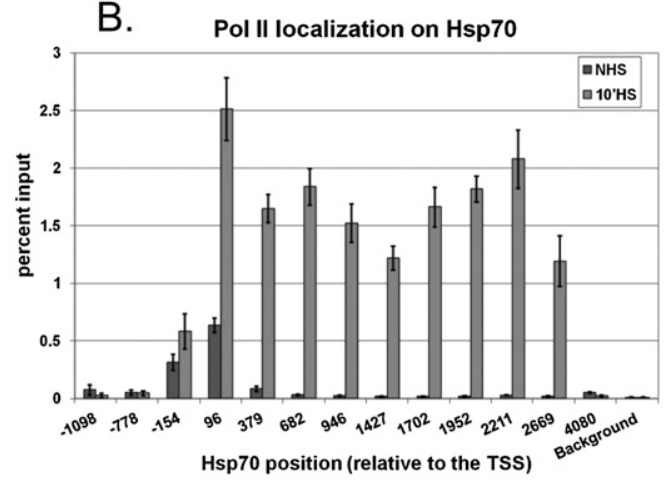

C.

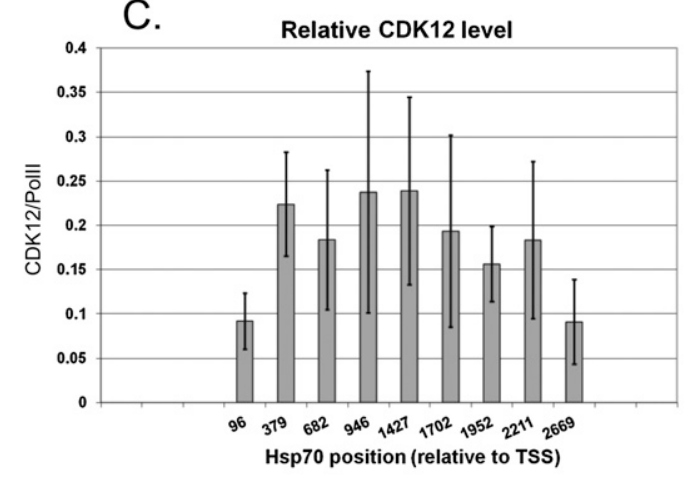

D.

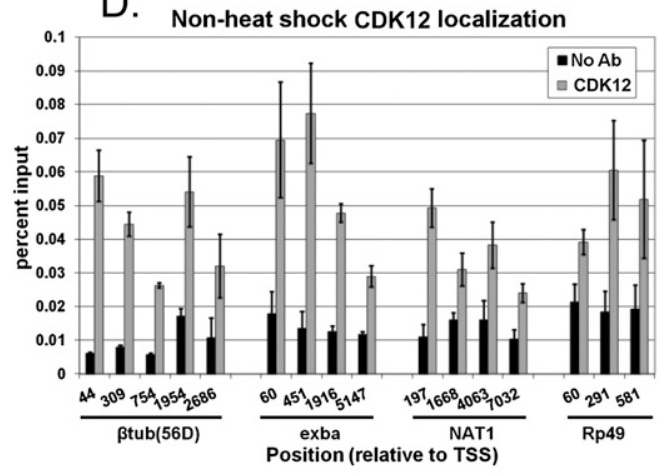

E. Non-heat shock Pol II localization

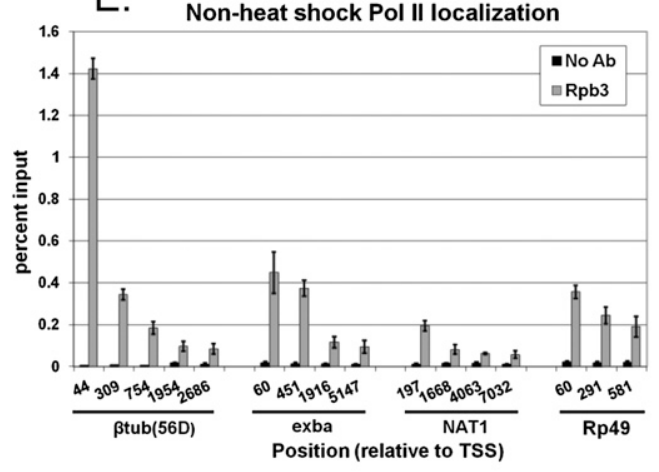

F.

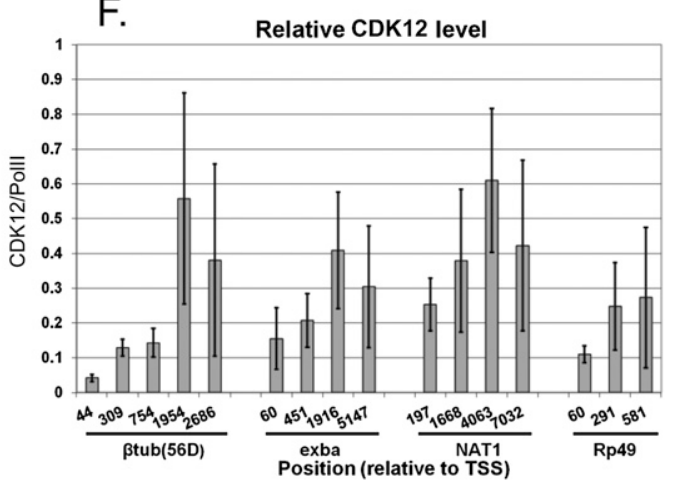

Figure 3. ChIP analysis of Pol II and dCDK12. (A) ChIP analysis of dCDK12 on Hsp70 using NHS and 10-min heat-shocked (10'HS) samples. (B) ChIP analysis of RNAPII (anti-Rpb3) on Hsp70, as in $A$. (C) Ratios of dCDK12/RNAPII values on 10-min heat-shocked Hsp70. (D) ChIP analysis of dCDK12 on four constitutively active genes under non-heat-shock conditions. (E) ChIP analysis of RNAPII (anti-Rpb3) on four constitutively active genes under non-heat-shock conditions. (F) Ratios of dCDK12/RNAPII values on four constitutively active genes under non-heat-shock conditions. Measurements in $A-F$ are averages of three biological replicates with standard errors.

Purified hCDK13 displayed CTD kinase activity in vitro when assayed using GST-yCTD fusion proteins as substrate (Fig. 4C). Upon hyperphosphorylation of the CTD by hCDK13 kinase, the migration rate of the fusion protein during SDS-PAGE is reduced, and a shifted band is generated in the autoradiogram (Fig. 4C, dotted line indicates position of unshifted substrate); this behavior mimics that of CTD substrates phosphorylated by yeast CTDK-I (Lee and Greenleaf 1989; Morris et al. 1997) and Drosophila CDK12 (Fig. 4A,B). Thus, our results demonstrate that hCDK13 manifests CTD kinase activity. While we have yet to similarly pull down and assay hCDK12, it has been reported in the literature that immunopurified hCDK12 (CrkRS) shows CTD kinase activity (Ko et al. 2001). Our assays of chimeric kinases (Supplemental Fig. S4C; data not shown) are consistent with this report.

\section{Cyclin component of metazoan CDK12 kinase}

When overexpressed in human cultured cells, tagged human CDK12 and CDK13 "pull down" overexpressed cyclins of the cyclin L family (Chen et al. 2006, 2007). To identify the cyclin normally associated with endogenous (not overexpressed) CDK12, we used Drosophila Kc cell 

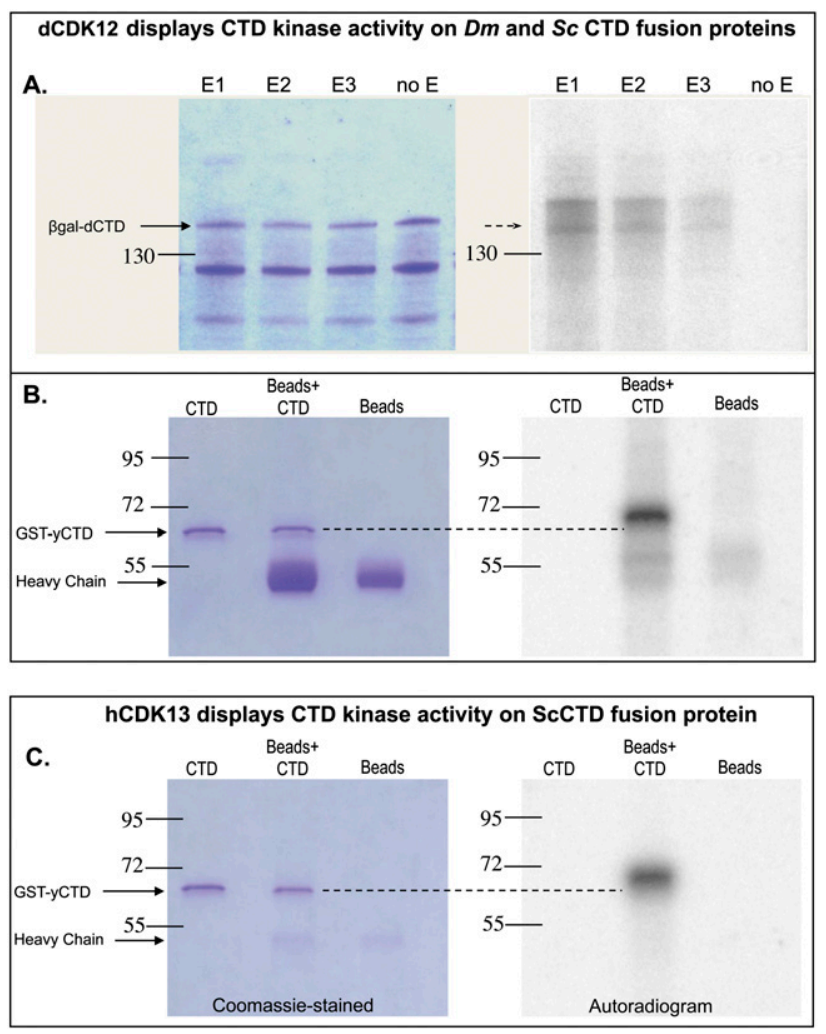

Figure 4. CTD kinase activities of isolated Drosophila dCDK12 or human hCDK13 enzymes. (A) dCDK12 was eluted from antibody beads using the antigenic peptide, and elution fractions (E1, E2, and E3) were assayed for kinase activity using a $\beta$ gal-dCTD fusion protein as substrate (apparent molecular weight $\sim 145 \mathrm{kDa}$ ). Coomassie-stained gel of terminated reactions is shown on the left, aligned with the corresponding autoradiogram on the right. (Lanes E1-E3) dCDK12 elutions all show CTD kinase (hyperphosphorylation) activity, as evidenced by the signals in the autoradiogram. No signal is observed in the no-enzyme control lane (fourth lane, no $E$ ). (B) dCDK12 was purified using protein $A / G$ beads saturated with affinity-purified anti-dCDK12 IgG. After extensive washing, including a $0.8 \mathrm{M}$ $\mathrm{NaCl}$ wash (see the Materials and Methods), the beads were assayed for CTD kinase activity using a GST-yCTD fusion protein as substrate. The Coomassie-stained gel on the left is aligned with the autoradiogram on the right. CTD kinase activity is observed (mobility-shifted band above GST-yCTD position) only when both beads (dCDK12) and CTD substrate are present. $(C)$ hCDK13 was isolated using ion-exchange chromatography followed by immunoaffinity purification (Materials and Methods); the antibody beads were washed extensively and assayed as in $B$. The mobility-shifted band (autoradiogram) above GST-yCTD position indicates hyperphosphorylation of CTD.

nuclear extract (Price et al. 1987). After an immunoaffinity purification of dCDK12 that included extensive washing, antibody bead-bound proteins were solubilized in SDS-containing buffer and subjected to SDS-PAGE. Staining with Coomassie blue revealed several obvious protein bands, in addition to a large amount of IgG heavy chain (Fig. 5). A major protein band (Fig. 5, arrow) migrated slower than the $130-\mathrm{kDa}$ marker and coincided with the major dCDK12 antibody-reactive band observed in Western blots (e.g., Supplemental Fig. S2). A gel slice containing this band ( $\mathrm{b}$ in Fig. 5) was excised and analyzed by mass spectrometry (LC MS/MS; Duke Proteomics Facility); the results confirmed the presence of dCDK12 in this gel slice (28 tryptic peptides identified, 33\% coverage of the protein, and "100\% probability" of correct identification). The dCDK12 protein probably migrates in SDS-PAGE slower than expected from its calculated molecular weight (128 kDa) due to the unusual amino acid composition of its $\mathrm{N}$-terminal extension.

Gel segments containing other bands (indicated in Fig. 5 as rectangular boxes a,c,d,e) were excised and similarly analyzed. We note first that no other CDK species (e.g., CDK9) were detected in any of the bands analyzed. Second, the only cyclin identified in this analysis was Cyclin K (in gel slice c; two peptides, but " $100 \%$ probability" of correct identification [Scaffold 2 Proteome software]). No peptides matching Cyclin L were detected in the analysis of the excised bands. Our results are therefore consistent with dCDK12 being associated with dCyclin K. Given the discrepancies among extant results (Fu et al. 1999; Chen et al. 2006, 2007), further experiments are warranted to test the idea that Cyclin $\mathrm{K}$ is the sole cyclin partner of CDK12/13.

\section{Chimeric human/yeast CTD kinases function in yeast}

Whereas the results presented to this point suggest that $\mathrm{dCDK} 12$ is orthologous to yCtk1, it has been suggested that, in higher eukaryotes, CDK9 reconstitutes the activities of both Bur1 and Ctk1 (Wood and Shilatifard 2006). To investigate this situation, we wanted to compare functional properties of hCDK9 with those of hCDK12/

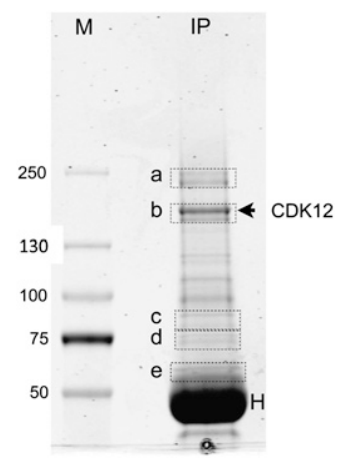

Figure 5. dCDK12 and copurifying proteins used for mass spectrometric protein identification. Antibody beads were used to isolate dCDK12 from a nuclear extract. After extensive washing of beads, proteins were solubilized in SDS buffer, subjected to SDS-PAGE, and stained with Coomassie blue. (M) Molecular weight marker lane (values at left); (IP) immunopurified protein lane. Arrow indicates band that is reactive with anti-dCDK12 antibodies. (Lanes $a-e$ ) Dotted lines indicate gel slices that were subjected to protein identification analysis in the Duke Proteomics facility. 
13 in yeast. However, because the very long $\mathrm{N}$-terminal and C-terminal extensions of hCDK12 and hCDK13 (Supplemental Fig. S3) are not like any protein in yeast (they contain many RS dipeptides and stretches of simple sequence), it seemed likely that the full-length human CDKs might cause dominant-negative effects or might be unstable in yeast. We therefore took the approach of constructing $C t k 1 / C D K x$ chimeric genes in which the central kinase homology region of CTK1 was excised and replaced with the corresponding piece of $h C D K 12$, $h C D K 13$, or $h C D K 9$; this generated chimeras $1 / 12,1 / 13$, and 1/9 (Supplemental Fig. S3). Two control chimeric constructs were also used: $1 / 1$ (i.e., wild-type CTK1) and $1 / 0$ (i.e., CTK1 deleted for the kinase homology region) (Supplemental Fig. S3). For analysis, these chimeric constructs were integrated into the genome by transformation to replace the endogenous CTK1 gene. Thus, each strain analyzed produced only a chimeric version of Ctk1.

With the chimeric kinase constructs, we could test for the ability of the human kinase homology domains (flanked by Ctk1 "arms") to rescue (suppress) phenotypes caused by mutation of CTK1 or BUR1. BUR1 is essential (CTK1 is not), and an extant burt ${ }^{\text {ts }}$ mutant (bur1-8) appeared to provide the best phenotype for use in the rescue experiments (good growth at $30^{\circ} \mathrm{C}$, but essentially no growth at $37^{\circ} \mathrm{C}$ ) (see Fig. 6, "+ empty vector" rows). (We note that, during the course of these studies, we observed that a phenotype often characteristic of $c t k 1 \Delta$ strains [cold sensitivity] is highly dependent on genetic background [data not shown]; therefore, we did not use this phenotype in our analysis.) Transforming the bur1-8 strain with plasmid-borne chimeric constructs, we tested the ability of the $1 / 9$ chimera and the $1 / 13$ chimera to

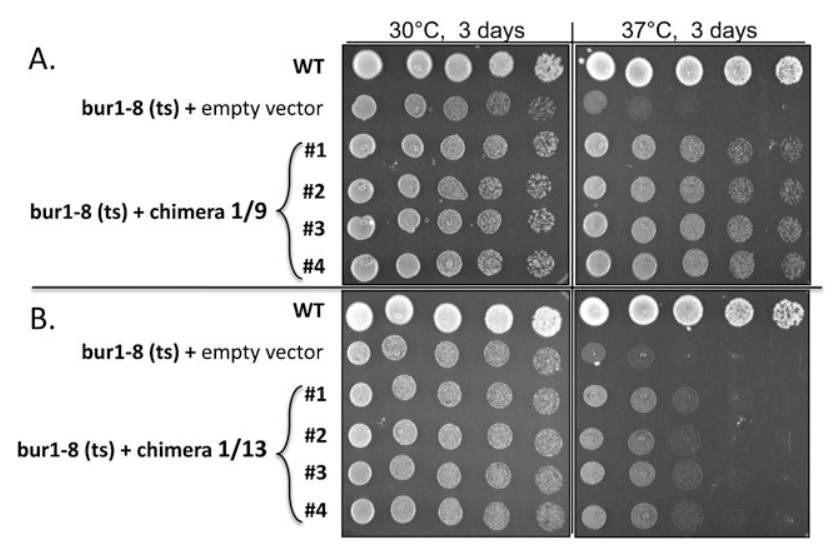

Figure 6. Complementation of bur1 ts allele by chimeric (yeast/human) kinases. (A) The bur1-8 (ts) yeast strain was transformed by empty vector or by vector carrying the $1 / 9$ chimeric kinase construct (cf. Supplemental Fig. S3), and independent transformants were tested for growth at $30^{\circ} \mathrm{C}$ and $37^{\circ} \mathrm{C}$ (fivefold serial dilutions spotted in rows). (WT) $B U R 1^{+}$ control strain. $(B)$ The bur1-8 (ts) strain was transformed by empty vector or by vector carrying the $1 / 13$ chimeric kinase construct, and independent transformants were tested for growth at $30^{\circ} \mathrm{C}$ and $37^{\circ} \mathrm{C}$ (fivefold serial dilutions spotted in rows). (WT) $B U R 1^{+}$control strain. rescue the temperature sensitivity caused by the bur1-8 allele. As shown in Figure 6A, four different transformants carrying the $1 / 9$ chimera grew well at the restrictive temperature of $37^{\circ} \mathrm{C}$. In contrast, four transformants carrying the $1 / 13$ chimera showed poor growth at $37^{\circ} \mathrm{C}$ (Fig. 6B). These results are consistent with the proposal that metazoan CDK9 is orthologous to yeast Bur1.

\section{Activities of natural and chimeric CTD kinases in vitro}

Since yeast strains containing chimeric Ctk1/12 or Ctk1/ 13 as the only Ctk1-type kinase grow well under laboratory conditions, we purified a chimeric enzyme from cells of such a strain for in vitro CTD kinase assays. As shown in Supplemental Figure S4A, the His-tagged chimeric $1 / 13$ subunit was accompanied by the endogenous Ctk3 subunit in fractions eluted from a $\mathrm{Ni}^{++}$column with imidazole. We infer that the Ctk2 (cyclin) subunit likewise copurified with the $1 / 13$ catalytic subunit (the kinase is active), but we could not test the inference directly due to our inability to detect Ctk2 in Western blots (cf., see Lee and Greenleaf 1991). CTD kinase assays (Supplemental Fig. S4B) confirm that the chimera phosphorylates a GST-yCTD fusion protein substrate, and that the amount of activity parallels the amount of enzyme (1/13 and Ctk3 subunits). We found virtually identical results with a $1 / 12$ chimera purified from a strain in which the only Ctk1 subunit was the $1 / 12$ chimeric protein (see assays in Supplemental Fig. S4C).

We also compared the CTDK-I, PTEF-b, hCDK13, Ctk1/12, and Ctk1/13 kinases on a set of CTD repeat sequence variants (Jones et al. 2004). As reported in Jones et al. (2004), yeast CTDK-I was about equally active (within a factor of 2) toward wild-type CTD, a CTD with each Ser2 replaced by Ala (A2), a CTD with each Ser2 replaced by Glu (E2), and a CTD with each Ser5 replaced with Glu (E5) (Supplemental Fig. S4C). In contrast, CTDK-I showed very low activity on a CTD with each Ser5 replaced by Ala (A5) (for interpretation, see Jones et al. 2004). With the same set of substrates, human P-TEFb displayed a similar specificity profile (data not shown). Furthermore, we found that hCDK13 (and the Ctk1/12 and Ctk1/13 chimeras) closely resemble yCTDK-I in their specificity profiles in vitro (Supplemental Fig. S4C for chimeras).

\section{Drosophila and human cells require CDK12 for normal phosphorylation of the CTD}

RNAi knockdown of dCDK12 in Drosophila cultured cells We used dsRNAs targeted at exon E2, E4, or E7 of dCDK12 to knock down the kinase in cultured S2 cells. Results of a 48-h experiment show that a high degree of dCDK12 depletion, relative to mock treatment, was achieved in each case (Fig. 7A, top panel). We analyzed the phosphorylation state of the CTD of Rpbl in each extract by Western blot analysis. In this analysis, Rpb1 with a nonphosphorylated CTD migrates in an SDS gel as band IIa, whereas Rpb1 with a highly phosphorylated CTD migrates more slowly, as band IIo (see Fig. 7F; also 


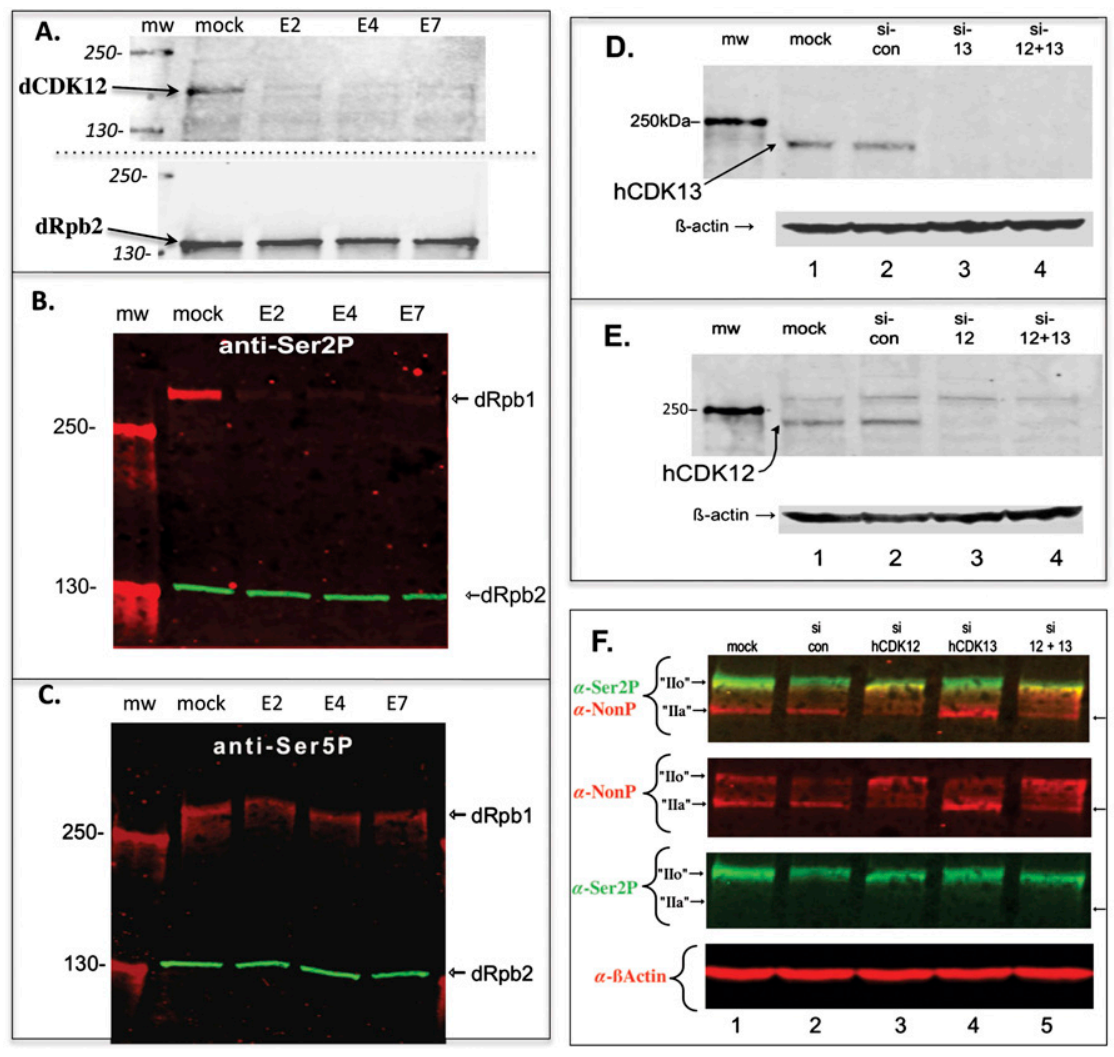
were performed as in $A$, extracts were prepared after $3 \mathrm{~d}$, and Western blot analysis was carried out using rAP anti-hCDK12 IgG and anti$\beta$-actin. $(F)$ Mock and siRNA treatments were performed on HeLa R-19 cells as in $A$, and Western blot analysis was carried out using rat antiSer2P (3E10) and mouse anti-non-phospho CTD (8WG16) mAbs. After analysis of Rpb1, the blot was reprobed with anti- $\beta$-actin antibodies. Note that the intensity of IIo in the si-control as detected by anti-Ser2P (lane 2) is usually the same as in the mock treatment (lane 1).

Greenleaf 1992). A critical point to keep in mind is that different patterns of CTD phosphorylation, as well as very different numbers of phosphates per CTD, can generate chemically distinct IIo species that migrate with very similar mobility in SDS gels (e.g., see Zhang and Corden 1991; Munoz et al. 2010). Probing with several antibodies is required to obtain even a partial picture of CTD phosphorylation status.

Figure $7 \mathrm{~B}$ shows that an anti-Ser2P mAb $(\mathrm{H} 5)$ produces a strong signal toward $\mathrm{dRpb} 1$ in the mock treatment sample (note, at the IIO position), whereas it produces a dramatically reduced signal in each of the RNAi-treated samples. An internal control, dRpb2, demonstrates very similar sample loading for all lanes (Fig. 7B). In contrast to Ser2P, Ser5P levels did not change significantly after RNA treatment, as monitored by anti-Ser5P mAb H14 (Fig. 7C). While differences between the Drosophila and human kinases and CTD primary sequences are considered in the Discussion, the results here show that dCDK12 is required for the majority of Ser2P (as detected by mAb H5) on Rpb1 in Drosophila whole-cell extracts.

RNAi knockdown of hCDK12 and hCDK13 in HeLa cells If hCDK12 and/or hCDK13 normally phosphorylate the CTD in vivo, then knockdown of one or both

Figure 7. RNAi knockdown of dCDK12, hCDK12, or hCDK13 in cultured cells. $(A)$ Mock and RNAi (dsRNA targeting exons $\mathrm{E} 2, \mathrm{E} 4$, or $\mathrm{E} 7$ of $d C D K 12=C G 7597)$ treatments were carried out for $48 \mathrm{~h}$ on cultured Drosophila S2 cells, extracts were prepared, and Western blot analysis was performed using rabbit affinity-purified anti-dCDK12 IgG. Goat affinity-purified anti-dRpb2 IgG was used to assess sample loading. Size standards are in the $m w$ lane, and values in kilodaltons indicated at left. (B) Mock and RNAi treatments and analysis were as in $A$, except that the phospho-CTD (PCTD) of dRpb1 was detected using mouse anti-Ser2P mAb (H5). Again, dRpb2 was a loading control. $(C)$ Mock and RNAi treatments and analysis were as in $B$, except that the PCTD of dRpbl was detected using mouse anti-Ser5P mAb (H14). (D) Mock and siRNA treatments were performed on HeLa R-19 cells, extracts were prepared after $3 \mathrm{~d}$, and Western blot analysis was carried out using rAP anti-hCDK13 IgG. The siRNAs used targeted hCDK13 (si-13), hCDK12 (si12), or both (si-12 + 13); control siRNAs (si-con) have no homology with any known mammalian genes. The $m w$ lane contains molecular weight markers, and the value is indicated at left. The blot was also reacted with anti- $\beta$-actin to show equal loading of with anti- $\beta$-actin to show equals. $(E)$ Mock and siRNA treatments ot blot analysis was carried out using rat anti- 
relative increase in reactivity of $\alpha$-NonP antibodies toward a slower-migrating Rpb1 band after knockdown of hCDK12 was not predicted; nevertheless, it is reproducible. One interpretation is that knockdown of hCDK12 increases the average number of nonphosphorylated repeats (or Ser5P-only repeats) per CTD, but CTD phosphorylation levels, on average, are nevertheless high enough to cause the majority of Rpb1 subunits to migrate at the IIo position (recall that both hCDK13 and hCDK9 are still active in these cells).

The anti-Ser2P signal intensity (Fig. 7F, green) after hCDK12 knockdown did not show the kind of decrease seen in the Drosophila dCDK12 knockdown experiment (Fig. 7B, red) (again, we stress that both hCDK13 and hCDK9 activities are still present). However, close inspection reveals that the $\alpha$-Ser2P band in the hCDK12 knockdown samples (Fig. 7F, lanes 3,5, green) appears slightly "sharper" than the corresponding band in the other lanes (the band's trailing edge appears to migrate slightly faster in Fig. 7F, lanes 3,5 than in lanes 1,2). Thus, the $\alpha$-Ser2P antibody does detect a difference in phosphoCTD (PCTD) state after hCDK12 knockdown. We note that the actual phosphorylation patterns determining the mobility of Rpb1 before and after knockdown are not discoverable by this approach. Overall, these experiments show that there is an observable change in the CTD phosphorylation state (as detected by both the $\alpha$-NonP and $\alpha$-Ser2P antibodies) upon hCDK12 knockdown.

Knockdown of hCDK13 also produced altered antibody reactivities that might suggest changes in CTD phosphorylation, although, compared with hCDK12, the changes were much more subtle. Frequently, we observed an apparent increase in the absolute amount of IIa signal, as seen in Figure 7F (red, lanes 1,2 vs. lane 4, internal standard: $\beta$-actin, red, bottom row). Thus, after knockdown of hCDK13, there appears to be an increase in amount of hypophosphorylated Rpb1, although a decrease in the anti-Ser2P signal was not observed (Fig. 7F, green, lane 4 vs. lanes 1,2).

These knockdown results are not unique to HeLa cells: Both hCDK12 and hCDK13 knockdown experiments were repeated in HEK293T/17 cells, with the same results (data not shown). Also, using $\alpha$-Ser5P mAb to probe the samples used in Figure 7F, we observed little obvious change in signal intensity or band migration after the knockdowns (reminiscent of the Drosophila $\alpha$-Ser5P results in Fig. 7C).

Overall, the data from the knockdown experiments, combined with other results, support the idea that hCDK12 is a bona fide CTD kinase in human cells. Because knockdown of hCDK13 does not appear to cause significant changes in global CTD phosphorylation, we cannot currently conclude that hCDK13 is a CTD kinase in vivo; however, in vitro, it clearly manifests CTD kinase activity (Fig. 4C).

\section{Discussion}

We show that fruit fly CDK12 and human CDK12 and CDK13 are CTD kinases. These proteins were identified previously by molecular evolution approaches as the closest Drosophila and human homologs of yeast Ctk1 (Liu and Kipreos 2000; Guo and Stiller 2004; cited in Phatnani and Greenleaf 2006), the major transcription elongation-associated CTD kinase in Sc. On the other hand, their status as functional CTD kinases was unclear. Our results demonstrate that dCDK12 is a major elongation-associated CTD kinase in Drosophila, the ortholog of yCtk1. Furthermore, our results support the proposal that, in human cells, hCDK12 is a functional counterpart of yCtk 1 ; hCDK13 is clearly a CTD kinase in vitro, but its functions in vivo are not yet clear. Because we also provide evidence that human CDK9 is orthologous to yeast Bur1 rather than yeast Ctk1, our findings help clarify the relationships between two yeast CDKs-Ctk1 and Burl-and their metazoan homologs. More importantly, our results draw attention to major metazoan CTD kinase activities that have gone unrecognized and unstudied until now.

In situ localizations of dCDK12 on Dm polytene chromosomes present a dramatic picture of the genomewide co-occurrence of this CTD kinase with hyperphosphorylated RNAPII. The dCDK12 kinase appears to be closely associated with Pol IIO, not only at loci expressed normally during development, but also at heat-shock loci (Fig. 1). In contrast, the chromosomal distributions of the two CTD kinases dCDK12 and dP-TEFb are distinct (Fig. 2). In addition, ChIP studies show that, whereas dP-TEFb amounts peak early in the gene (basically, with paused polymerase), dCDK12 amounts peak later in the transcription unit (Fig. 3), resembling the behavior of yCtk1. Because functions of P-TEFb, such as helping RNAPII to overcome an impediment to elongation early in transcription (Peterlin and Price 2006), are presumably reflected in its site-specific localization patterns, we propose that the chromosomal localization patterns of dCDK12 similarly reflect its functions during transcription. That dCDK12 displays CTD kinase activity in vitro supports the idea that the principal function reflected in its chromosomal distribution is, in fact, phosphorylation of the CTD on elongating RNAPII. CTD phosphorylation on elongating RNAPII is also the most likely function of the human homolog hCDK12, making it a probable ortholog of yCtk1. While the in vivo function of hCDK13 is not yet clear, our genetic experiments place both human paralogs-hCDK12 and hCDK13-in a functional group related to yeast Ctk1. In contrast, the genetic experiments place the other human elongation-associated CTD kinase, $\mathrm{CDK} 9$, in a different functional group related to yeast Burl.

Knockdowns of dCDK12 and hCDK12 support the proposal that these kinases phosphorylate the CTD of Rpb1 in vivo. Even looking at total RNAPII in whole-cell extracts of human cells, we observe a change in CTD phosphorylation state after hCDK12 knockdown (Fig. 7C). As argued elsewhere (Phatnani and Greenleaf 2006; Munoz et al. 2010), it is impossible to obtain a detailed understanding of phosphorylation patterns from antibody reactivities, but, in the current context, the important finding is that knockdown of hCDK12 results in alteration of phosphorylation status for the CTD of RPB1. The 
relative increase in slower-migrating RPB1 species after knockdown of hCDK12 (Fig. 7) seems counterintuitive, but it may not be coincidental that an analogous observation was made previously in $c t k 1 \Delta$ strains of yeast (Patturajan et al. 1999). Also, a knockdown of one human paralog (e.g., hCDK12) leaves the other (hCDK13) still active in the cells. In Drosophila cells, which contain only one Ctk1 ortholog, knockdown results are more apparent; over two-thirds of Ser2 phosphorylation, as defined by the $\mathrm{mAb} \mathrm{H} 5$, is lost after knockdown of dCDK12 (Fig. 7E). In addition to there being only a single Ctk1-type kinase in Drosophila cells, one set of potential explanations for this dramatic effect (as compared with the more subtle global CTD phosphorylation changes in the mammalian cell knockdowns) lies in the unusually low occurrence of consensus heptad repeats in the dCTD (two out of 45 vs. 21 out of 52 in mammals) (Corden 1990). For example, the $\mathrm{H} 5 \mathrm{mAb}$ might detect almost all of the Ser2P residues in the mammalian CTD, but only a subset of Ser2P residues in the $\mathrm{dCTD}-$ one that is produced mainly by dCDK12. Other potential explanations include those that invoke additional differences (known or unknown) between flies and mammals, such as the extent of functional redundancy between CDK12/13 and CDK9 kinases. Independent of which explanation is correct, our overall results make a compelling case for the proposal that dCDK12 and hCDK12 function as CTD kinases in vivo.

It has been argued in the literature that the cyclin partners of hCDK12 and hCDK13 are members of the Cyclin L family (Chen et al. 2006, 2007). However, our results are consistent with the idea that Cyclin $\mathrm{K}$ is the actual partner of dCDK12 (and, presumably, hCDK12/13 as well). Because the cyclin partner of $S c \mathrm{Ctk} 1$ is Ctk2, we used BLAST to find sequences most similar to Ctk2 under "metazoa" in the NCBI nonredundant protein database; the top-scoring hits were all Cyclin $\mathrm{K}$. In addition, a yeast two-hybrid screen using dCDK12 (CG7597) picked up dCyclin $\mathrm{K}$ with the highest score among seven positives (Finley Laboratory YTH results in the Drosophila Interactions Database, http://www.droidb.org). Thus, evidence favoring Cyclin $\mathrm{K}$ as the physiological partner of CDK12 is accumulating. Interestingly, Cyclin K was found previously to associate with CDK9 and to stimulate its CTD kinase activity in vitro (Fu et al. 1999). Future analyses will be required to substantiate the partnership of cyclin K with CDK12 (and CDK13), and to determine whether Cyclin $\mathrm{K}$ functionally interacts with both CDK12/13 and CDK9 in vivo.

Another feature attributed to CDK12/13 is a direct involvement in specific splice site choices (Chen et al. 2007; Berro et al. 2008). However, we predict that, as for Ctk1 in yeast (Phatnani et al. 2004), knocking down expression or activity of "metazoan Ctk1" (e.g., dCDK12 in Drosophila cells) will lead to abnormalities in splicing indirectly as a result of anomalous CTD phosphorylation and attendant defects in recruitment of splicing factors to elongating RNAPII (cf. Greenleaf 1993; Goldstrohm et al. 2001). The presence in human cells of two similar but functionally divergent kinases makes analogous predic- tions for hCDK12 or hCDK13 moot until the enzymes have been characterized more thoroughly.

In general, we expect that any process or event dependent on normal phosphorylation of the CTD on elongating RNAPII will be affected in cells deficient for CDK12 (and possibly CDK13). In addition to splicing, such events include other kinds of RNA processing, such as small RNA termination (e.g., see Steinmetz et al. 2001; Kim et al. 2006), pre-mRNA $3^{\prime}$ end formation (e.g., Bentley 2005), and probably mRNA export out of the nucleus (A MacKellar and AL Greenleaf, unpubl.). In addition, normal transcription-coupled chromatin modification depends on CTD phosphorylation by yCTDK-I (e.g., see Kizer et al. 2005). Finally, alterations in transcription-associated homologous recombination events have been observed in ctk14 strains (Grenetier et al. 2006; Westmoreland et al. 2009), and certain proteins important to genome stability are linked to transcription via direct interaction with the hyperphosphorylated CTD (Islam et al. 2010; Kanagaraj et al. 2010) (T Winsor, C Bennett, and AL Greenleaf, in prep.); mice missing one of these PCTD-associating genome stability proteins (RECQ5) exhibit an increased incidence of cancer (Hu et al. 2007). Thus, we anticipate that further characterizations of CDK12 and CDK13 will be of broad scientific interest and significant medical relevance.

\section{Materials and methods}

\section{Antibodies and Western blot analysis}

Anti -IIo: goat affinity-purified IgGs directed toward the hyperphosphorylated CTD of DmRpb1 (Weeks et al. 1993; Morris et al. 1997). rAP $\alpha$-dCDK12: rabbit affinity-purified IgGs directed against a peptide comprising amino acids 705-722 of dCDK12 (CG7597; NP_649325.2). rAP $\alpha$-hCDK13: rabbit affinity-purified IgGs directed against a peptide comprising amino acids 294312 of hCDK13 (CDC2L5. NCBI RefSeq: NP_003709.3). rAP $\alpha$-HCDK12: rabbit affinity-purified IgGs directed against a peptide comprising amino acids 1058-1077 of hCDK12 (CRKRS. NCBI RefSeq: NP_057591.2). Anti-dCyclin T: affinity-purified sheep IgG against purified dCyclin T. Anti-NonP: mAb 8WG16 (Abcam). Anti-Ser2P: rat monoclonal (3E10) from D. Eick (Chapman et al. 2007), or mouse $\mathrm{mAb}(\mathrm{H} 5)$ from Covance in Figure 7E. Anti-Ser5P: mouse mAb (H14) from Covance. Antibody to $\beta$-actin, a mouse mAb from Abcam. Anti-HA: mouse monoclonal 12CA5 from Roche. Anti-Ctk3: affinity-purified rabbit anti-yCtk3 antibody (Sterner et al. 1995). gAP-anti-dRpb2: affinity-purified goat IgG against a segment from dRpb2 (amino acids 519-992) (Skantar and Greenleaf 1995).

Western blot analysis was performed using the Odyssey infrared scanner and secondary antibodies from Li-Cor.

\section{Microscopy}

Visualization of proteins on fixed polytene chromosomes (Greenleaf et al. 1976; Weeks et al. 1993) was carried out as described previously (Schwartz et al. 2004).

\section{ChIP}

Drosophila S2 cells were grown in M3 + BPYE with 10\% FBS to a density of $6 \times 10^{6}$ to $7 \times 10^{6}$ cells per milliliter. To prepare the 
10-min heat-shock chromatin, $4 \mathrm{~mL}$ of $48^{\circ} \mathrm{C} \mathrm{M} 3+$ BPYE (no serum) was added to $4 \mathrm{~mL}$ of culture and incubated for $10 \mathrm{~min}$ at $36.5^{\circ} \mathrm{C}$, and then $4 \mathrm{~mL}$ of $4^{\circ} \mathrm{C} \mathrm{M} 3+\mathrm{BPYE}$ (no serum) was added to bring the cells to room temperature. Immediately, formaldehyde was added to a final concentration of $1 \%$ and mixed for 2 min at room temperature. The reaction was quenched with $675 \mu \mathrm{L}$ of $2.5 \mathrm{M}$ glycine and immediately cooled on ice. The cells were centrifuged for $5 \mathrm{~min}$ at $4^{\circ} \mathrm{C}$ and resuspended in sonication buffer (20 mM Tris-Cl at $\mathrm{pH}$ 8.0, $2 \mathrm{mM}$ EDTA, $0.5 \mathrm{mM}$ EGTA, 0.5\% SDS, 0.5 mM PMSF, Roche complete protease inhibitor cocktail [catalog no. 05056489001 ]) at $10^{8}$ cells per milliliter. The cells were sonicated 12 times for $20 \mathrm{sec}$ each time with a 1-min rest in between at $4^{\circ} \mathrm{C}$ using a Bioruptor sonicator; the material was centrifuged at $20,000 \mathrm{~g}$ for $10 \mathrm{~min}$ at $4^{\circ} \mathrm{C}$, and $25 \mu \mathrm{L}$ of the supernatant was used per immunoprecipitation.

Pol II was immunoprecipitated with $4 \mu \mathrm{L}$ of rabbit anti-Rpb3 antisera (Lis laboratory stock), HSF was immunoprecipitated with $4 \mu \mathrm{L}$ of rabbit anti-HSF antisera (Lis laboratory stock), and dCDK12 was immunoprecipitated with $7 \mu \mathrm{L}$ of $\mathrm{rAP} \alpha$-dCDK12. The non-heat-shock chromatin was prepared in the same manner, except $8 \mathrm{~mL}$ of room temperature M3 + BPYE (no serum) was added to $4 \mathrm{~mL}$ of culture. The amount of immunoprecipitated DNA was quantified using a Roche LightCycler 480 by comparing the quantification cycle of the immunoprecipitated DNA to the quantification cycle of serial dilutions of input DNA to obtain the percent input.

\section{CTD kinase assays}

CTD kinase assays were performed essentially as described (Jones et al. 2004), with minor variations (e.g., total [ATP] was typically $60-100 \mu \mathrm{M}$ ).

\section{Mass spectrometry}

Fifteen microliters of dCDK12 bead slurry (see dCDK12 purification) was subjected to PAGE using a $4 \%-15 \%$ SDS gel. After staining with Coomassie blue, gel bands of interest were excised and submitted for LC MS/MS analysis at the Duke Proteomics Facility (http://www.genome.duke.edu/cores/proteomics).

\section{Kinase purifications}

dCDK12 purification Two milliliters of $\mathrm{KcN}$ extract (Price et al. 1987) were diluted to a final volume of $4 \mathrm{~mL}$ in ice-cold HGEDPF (25 mM HEPES, 8\% glycerol, $0.1 \mathrm{mM}$ EDTA, $1 \mathrm{mM}$ DTT, phenylmethylsulfonyl fluoride [1:1000 dilution of saturated solution in isopropanol], $10 \mathrm{mM} \mathrm{NaF}$; $\mathrm{NaCl}$ and Triton $\mathrm{X}-100$ were added to a final concentration of $0.5 \mathrm{M} \mathrm{NaCl}$ and $0.4 \%$ Triton $\mathrm{X}-100$. The extract was then centrifuged at $23,000 \mathrm{~g}$ for $1 \mathrm{~h}$ at $4^{\circ} \mathrm{C}$, and the supernatant was precleared by rocking end-over-end with $20 \mu \mathrm{L}$ of protein A/G bead slurry (Thermo Scientific) for $1 \mathrm{~h}$ at $4^{\circ} \mathrm{C}$. Approximately $50 \mu \mathrm{L}$ of protein A/G beads (Thermo Scientific, Ultra-Link resin) were saturated with $\mathrm{rAP} \alpha-\mathrm{dCDK} 12 \mathrm{IgG}$ and added to the precleared extract, and then allowed to rock end-over-end overnight at $4^{\circ} \mathrm{C}$. The beads were recovered, transferred to a small disposable column (Bio-Rad), and washed at $4^{\circ} \mathrm{C}$ with $10 \mathrm{~mL}$ of PBS $+0.2 \%$ Tween 20 , then with $10 \mathrm{~mL}$ of HGEDPF at $0.5 \mathrm{M} \mathrm{NaCl}$, followed by another 10 $\mathrm{mL}$ of PBS $+0.2 \%$ Tween 20 . The beads were then transferred to a fresh microfuge tube and resuspended in $100 \mathrm{mM} \mathrm{NaCl}$ / HGEDPF. Beads used directly for kinase assays were further washed in $0.8 \mathrm{M} \mathrm{NaCl} / \mathrm{HGEDPF}$ before being rinsed and resuspended in $1 \times$ kinase buffer (25 mM HEPES at pH 7.6, $10 \mathrm{mM}$ $\mathrm{MgCl}_{2}, 5 \mathrm{mM} \mathrm{NaF}$ ).
dCDK12 peptide elution Protein A acrylic beads (Sigma, cata$\log$ no. P1052) were saturated with dCDK12 antibody and $100 \mu \mathrm{L}$ of beads incubated with $2.5 \mathrm{~mL}$ of $\mathrm{KcN}$ extract for $2 \mathrm{~h}$ at $4^{\circ} \mathrm{C}$ with end-over-end rocking in a Bio-Rad disposable column. The beads were washed in HGEDP (same as HGEDPF, but without $\mathrm{NaF}$ ) with $0.5 \% \mathrm{NP}-40$ and $100 \mathrm{mM} \mathrm{KCl}$, followed by $400 \mathrm{mM} \mathrm{KCl} /$ HGEDP and $100 \mathrm{mM} \mathrm{KCL/HGEDP} \mathrm{without} \mathrm{detergent.} \mathrm{Elution}$ 1 was carried out by a $1-h$ incubation with end-over-end rocking at $4^{\circ} \mathrm{C}$ with $1 \mathrm{mM}$ antigenic peptide (ABR/Thermo Scientific) in $100 \mu \mathrm{L}$ of $100 \mathrm{mM} \mathrm{KCl} /$ HGEDP. Elutions 2 and 3 were performed in the same manner, but with a concentration of $100 \mu \mathrm{M}$ antigenic peptide. For assays, $5 \mu \mathrm{L}$ of each elution was used.

hCDK13 purification HeLa nuclei were prepared, extracted with $0.15 \mathrm{M} \mathrm{NaCl}$, and centrifuged (Longley et al. 1997). Twenty-five milliliters of the pellet was used to prepare a $0.5 \mathrm{M} \mathrm{NaCl}$ extract of the nuclear pellet as described previously (Carty et al. 2000). The $0.5 \mathrm{M} \mathrm{NaCl}$ extract was filtered through Miracloth (Chicopee Mills, Inc.) to remove floating lipid, and protein concentration was determined using the Bradford method (Bio-Rad Protein Assay). Ion exchange chromatography was performed on a 25-mL Macro-Prep CM (Bio-Rad) column. Extract containing $400 \mathrm{mg}$ of protein was diluted to $0.15 \mathrm{M} \mathrm{NaCl}$ with HGEDPF and batch-bound to the equilibrated Macro-Prep $\mathrm{CM}$ slurry for $2 \mathrm{~h}$ at $4^{\circ} \mathrm{C}$. The slurry was then loaded back into the column, washed with $0.15 \mathrm{M} \mathrm{NaCl} / \mathrm{HGEDPF}$, and eluted using steps of $0.25,0.4,0.6$, and $1 \mathrm{M} \mathrm{NaCl}$ in HGEDPF. The bulk of hCDK13 was found to elute in the $0.6 \mathrm{M} \mathrm{NaCl}$ fraction (monitored by Western blot). The $0.6 \mathrm{M}$ fractions containing hCDK13 were pooled and frozen at $-80^{\circ} \mathrm{C}$ in $1-\mathrm{mL}$ aliquots.

Antibody-saturated beads prepared with Ultra-Link protein A/G beads (10-100 $\mu \mathrm{L}$ of slurry) or magnetic protein G Dynabeads (50 $\mu \mathrm{L}$ of slurry) and $\mathrm{rAP} \alpha$-hCDK13 were mixed with the $0.6 \mathrm{M} \mathrm{NaCl} \mathrm{CM}$ column fractions $(1-8 \mathrm{~mL})$ and allowed to rock end-over-end for $8 \mathrm{~h}$ to overnight. The beads were then washed with multiple bead volumes for each of the following: PBS $/ 0.2 \%$ Tween20, $0.8 \mathrm{M} \mathrm{NaCl} / \mathrm{HGEDPF}$, PBS/0.2\% Tween20, and, finally, $1 \times$ kinase reaction buffer $(25 \mathrm{mM}$ HEPES at $\mathrm{pH} 7.6,10 \mathrm{mM}$ $\mathrm{MgCl}_{2}, 5 \mathrm{mM} \mathrm{NaF}$ ). The beads were resuspended in $1 \times$ kinase reaction buffer, frozen in liquid nitrogen, and stored at $-80^{\circ} \mathrm{C}$.

Chimeric kinase purification An FY23 strain bearing Ctk1/13 was grown in $6 \mathrm{~L}$ of $\mathrm{YPD}$ at $30^{\circ} \mathrm{C}$ to an $\mathrm{OD}_{600}$ of 1 , and cells were collected by centrifugation and washed with ice-cold buffer $\mathrm{A}$ (50 mM HEPES, $100 \mathrm{mM} \mathrm{NaCl}$ ), then spun at $2600 \mathrm{~g}$ for $10 \mathrm{~min}$ at $4^{\circ} \mathrm{C}$. The pellet was pushed through a syringe into liquid nitrogen to make frozen "noodles" (Oeffinger et al. 2007), which were stored at $-80^{\circ} \mathrm{C}$. The cell noodles were homogenized in the frozen state using a Mixer Mill MM400 (Retsch) (Oeffinger et al. 2007). The ground yeast powder was resuspended in buffer (50 mM HEPES at $\mathrm{pH} 7.6,0.55 \mathrm{M} \mathrm{NaCl}, 15 \%$ glycerol, $0.05 \%$ Triton X-100, $25 \mathrm{mM}$ $\beta$-glycerol-phosphate, $10 \mathrm{mM} \mathrm{NaF}$, plus, just before use, PMSF, DTT, protease inhibitor cocktail, phosphatase inhibitor cocktail) by rotating end-over-end for $10 \mathrm{~min}$ and homogenizing with a glass dounce to generate a uniform suspension (this and all subsequent steps were at $4^{\circ} \mathrm{C}$ ). The suspension was centrifuged for $40 \mathrm{~min}$ at $26,000 \mathrm{~g}$, and the supernatant was passed through a 2.7-um syringe filter (Whatman GF/D). After adjusting the conductivity to $400 \mathrm{mM} \mathrm{NaCl}$ equivalent, the clarified extract was passed through a DE52 column (equilibrated at $400 \mathrm{mM} \mathrm{NaCl}$ ) and the flow-through fraction was collected; $0.27 \mathrm{~g} / \mathrm{mL}(\mathrm{NH} 4)_{2} \mathrm{SO}_{4}$ was added slowly with gentle stirring until dissolved, and stirring continued for another $30 \mathrm{~min}$. Precipitated proteins were collected by centrifugation at $26,000 \mathrm{~g}$, and the pellet was resuspended in buffer HGE (25 mM HEPES at pH 7.6, 15\% glycerol, 0.1 mM EDTA). 
The protein mixture was adjusted to equal to $100 \mathrm{mM} \mathrm{NaCl}$ conductivity and mixed with Macro-Prep CM resin. Elutions were made with salt steps of $200 \mathrm{mM} \mathrm{NaCl}, 400 \mathrm{mM} \mathrm{NaCl}$, and $600 \mathrm{mM} \mathrm{NaCl}$ in HGE buffer, and peak kinase fractions were identified by Western blot (C-terminal HA tag) or kinase assay and pooled. Pooled kinase was applied to a Ni-Sepharose6 Fast Flow column (GE Healthcare) and eluted with Ni elution buffer $(50 \mathrm{mM}$ Tris at $\mathrm{pH} 8.0,100 \mathrm{mM} \mathrm{NaCl}, 250 \mathrm{mM}$ imidazole, $15 \%$ glycerol). Kinase proteins were detected by Western blotting (anti-HA tag for Ctk1/X, and anti-Ctk3 for Ctk3) (Sterner et al. 1995). The $1 / 12$ chimera was purified by the same approach.

\section{Yeast strains and genetics}

Yeast strains, DNA plasmids, and PCR primers are listed in the Supplemental Material.

All yeast transformations were done according to standard LiOAc transformation methods.

\section{GY2002 bur1-8 ts yeast complementation assay}

Yeast strain GY2002 was a gift from G. Prelich. Four different GY2002 colonies were verified for their ts phenotype at $37^{\circ} \mathrm{C}$, and one was used in the following complementation assay. GY2002 yeast cells were transformed with pAG415GALCTK1/X-TRP1-LEU2 plasmid or empty pAG415GAL-TRP1LEU2 plasmid and selected on CM-Trp plates. Colonies were grown and extracted DNA was sequenced to validate the plasmid. GY2002 cells transformed with different chimeric constructs, as well as a negative control with only the empty vector and a positive control, which is yeast strain $\mathrm{Z} 26\left(T R P 1^{+}\right.$, $L E U 2^{+}$), were grown to $\mathrm{OD}_{600}$ of 1.0 in CM-Trp at $30^{\circ} \mathrm{C}$ with shaking, and were spotted on CM-Leu plates in 1:5 serial dilutions. Replica plates were placed for $3 \mathrm{~d}$ at $30^{\circ} \mathrm{C}$ or $37^{\circ} \mathrm{C}$ before analysis.

\section{Cloning (chimeras)}

Construction of CTK1/13, CTK1/12, CTK1/9, and CTK1/0 chimeric kinases plasmids with TRP1 yeast marker A pUC19based plasmid pVD45 containing 3.8-kb CTK1-HA3::K.I.TRP1 (which contains CTK1 $5^{\prime}$ and $3^{\prime}$ flanking homology sequence, CTK1 endogenous promoter, CTK1 gene with C terminus tagged by $3 \mathrm{HA}$ tags, and a yeast TRP1 marker) was obtained from Peter V. Decker in the Greenleaf laboratory (see the Supplemental Material). To replace the CTK1 kinase domain (amino acids 183393) with corresponding catalytic regions from hCDK13, hCDK12, and hCDK9, both the $\mathrm{N}$-terminal and C-terminal parts of the CTK1 sequence flanking the kinase domain were PCR-amplified and cloned into pGEX-5X-1 (Amersham). CTK1 NF and NR primer pair was used to introduce EcoNI and BamHI at both ends of the CTK1 N-terminal sequence, and these two RE sites were used to clone CTK1N into pGEX-5X-1. Later, CTK1C PCR generated by CTK1 CF and CR pairs was ligated into pGEXCTK1N via BamHI and XhoI sites included in the primer sequence to create pGEX-CTK1NC. hCDK13, hCDK12, and hCDK9 kinase domains were PCR-amplified, and BamHI sites were introduced at both ends by CDK13 F and R, CDK12 F and R, and CDK9 $\mathrm{F}$ and $\mathrm{R}$ from pCMV-SPORT6-CDC2L5 vector (Open Biosystems), pCMV6-XL-CDK12 plasmid (Origene), and pCMVSPORT6-CDK9 vector (Open Biosystems), respectively. All of the human kinase domain PCR products were cloned into pGEXCTK1NC via BamHI sites to generate plasmid pGEX-CTK1/X, and directional cloning was sequence-verified. DNA fragments containing the chimeric kinases (CTK1/13, CTK1/12, and CTK1/9) were cut out by PmlI and MluI, retained in the natural
CTK1 upstream and downstream sequence, and cloned into pVD45 vector to replace the wild-type CTK1 sequence. For CTK1/0 construction, a PmlI and MluI fragment from pGEXCTK1NC was cloned into pVD45 plasmid. Two complementary oligonucleotides coding for His6 tag (His6 MluI F and R) were annealed and cloned to the $\mathrm{C}$ terminus of the chimeric kinases by MluI sites in the middle of the 3HA tags, leaving two HA tags intact. Similarly, five strep-tactin tags (coded by oligonucleotides Strep BstPI $\mathrm{F}$ and $\mathrm{R}$ ) were added at the $\mathrm{N}$ terminus of the chimeric kinases via BstPI sites to facilitate the kinase purification. All of the pVD45 chimeric kinase constructs (CTK1/ 13, CTK1/12, CTK1/9, and CTK1/0) were sequence-verified. SacI and Sall fragments containing the $5^{\prime}$ and $3^{\prime}$ CTK1 homologous sequence, CTK1/X chimeric kinase sequence, and TRP1 marker were used to transform FY23 yeast strains to replace the endogenous wild-type CTK1 gene.

Construction of CTK1/13, CTK1/12, CTK1/9, and CTK1/0 chimeric kinase plasmids with URA3 yeast marker NotI and SapI fragments from pVD62 plasmid (from Peter V. Decker in the Greenleaf laboratory) (Supplemental Material) containing a URA3 gene were cloned into pVD45-CTK1/X plasmids with the same RE sites to replace the TRP1 markers, generating a series of pVD62-CTK1/X-URA3 plasmids whose SacI and SalI DNA fragments were used in BY4742 bre $1 \Delta$ and bre $2 \Delta$ transformation assays.

Construction of CTK1/13, CTK1/9, and CTK1/0 centromeric plasmids with LEU2 yeast marker About $3.8 \mathrm{~kb}$ of DNA fragments were PCR-amplified from $\mathrm{pVD} 45-C T K 1 / X$ constructs by primer pair YTpVD45 $\mathrm{F}$ and $\mathrm{R}$ with High-Fidelity Taq (Invitrogen) to cover the endogenous CTK1 promoter and CTK1/X sequence. The PCR products were ligated into PCR8/ $\mathrm{GW} / \mathrm{TOPO}$ vector (Invitrogen) via the method provided in the manual. The sequencing-verified directional clone was incubated with Gateway destination vector pAG415GAL-ccdB$L E U 2$ via LR reaction, and the resulting pAG415GAL-CTK1/ $X$-TRP1-LEU2 constructs were used in the bur1 ts complementation assays.

\section{Cell culture and RNAi}

Human cells The HeLa R-19 cell line was a kind gift from Dr. Mariano Garcia-Blanco. HeLa R-19 was cultured in DMEM with $10 \%$ FBS and $1 \times$ PenStrep (all from GIBCO). Experimentally verified FlexiTube siRNAs targeting hCDK13 (Hs_CDC2L5_5 and Hs_CDC2L5_6) and hCDK12 (Hs_CRK7_5 and Hs_CRK7_6) were purchased from Qiagen. The control siRNA is AllStars Negative Control siRNA from Qiagen. HeLa R-19 cells were reverse-transfected in six-well plates and/or 60-mm dishes using RNAi Max (Invitrogen) transfection reagent following the manufacturer's instructions. One siRNA targeting hCDK12 (Hs CRK7_5) was found to have an off-target effect that resulted in cell death, and was not used. The second hCDK12 siRNA and a pool of the two hCDK13 siRNAs were used for the knockdown experiments. Knockdown experiments were conducted with seeding densities calculated so that the cells reach $\sim 90 \%$ confluence before being harvested. Final siRNA concentrations of $5 \mathrm{nM}$ and $12.5 \mathrm{nM}$ (total concentrations for both the pooled [CDK13] and single [CDK12] siRNAs) and time points of 48 and $72 \mathrm{~h}$ were found to yield the same results. Cells were harvested by trypsinization with $0.05 \%$ Trypsin-EDTA (GIBCO) for $3 \mathrm{~min}$ at $37^{\circ} \mathrm{C}$, followed by neutralization of the trypsin with complete medium. The resulting cell slurry was centrifuged at low speed, and the cell pellet was washed by resuspension in $5 \mathrm{~mL}$ of PBS. The cells were pelleted, resuspended in $1 \mathrm{~mL}$ of PBS, and 
repelleted. After removing the supernatant, the cell pellet was lysed with RIPA buffer or hot $2 \times$ SDS loading buffer (final volume $\sim 130 \mu \mathrm{L}$ ). Successful knockdown of the kinases was confirmed by Western blot analysis.

Drosophila cells Constructs targeting CG7597 were designed using the SnapDragon program (http://www.flyrnai.org/cgi-bin/ RNAi_find_primers.pl), which is optimized for the design of long dsRNAs for $D m$. Three constructs were selected (targeting exon 2, exon 4, and exon 7 of CG7597), and the primers were extended with the sequence of the $\mathrm{T} 7$ promoter.

\section{Constructs}

Constructs used were as follows: E2F, 5'-GAATTAATACGA CTCACTATAGGGAATCAATGCCTTACTGGACCG-3'; E2R, 5'-GAATTAATACGACTCACTATAGGGATGGTATCGTGACT GCTCGAC-3'; E4F, 5'-GAATTAATACGACTCACTATAGGGA GTGTGCAGCACTCACGAAGT-3'; E4R, 5'-GAATTAATACG ACTCACTATAGGGACTCTTGCCGCTCAATGATTT-3'; E7F, 5'-GAATTAATACGACTCACTATAGGGACTCCTACGGTGAT AGCCTCG-3'; E7R, 5' -GAATTAATACGACTCACTATAGGGA ATCTGATGCTGTGTGCGTTC-3'.

The primers were used to amplify DNA from Drosophila genomic DNA (a gift from Dr. Tao Hsieh's laboratory). dsRNA was made using a MEGAScript T7 Kit (Ambion, Inc.) following the manufacturer's instructions; the resulting RNA was purified using phenol chloroform extraction and annealed. S2 cells were grown in M3 + BPYE with $10 \%$ serum. Cells $\left(1.5 \mathrm{~mL}\right.$ of $1 \times 10^{6}$ cells per milliliter) were silenced using $15 \mu \mathrm{g}$ of dsRNA in a sixwell plate format for $48 \mathrm{~h}$. Anti-LacZ dsRNA was used as a control.

\section{Acknowledgments}

We thank Craig B. Bennett and April MacKellar for insights and helpful discussions, Janis Werner for performing the polytene staining experiments, Daniel A. Gilchrist for an introduction to S2 cells, Dirk Eick for the kind gift of mAb 3E10, and Greg Prelich for the bur1-8 strain. This research was supported by grants from the NIH to A.L.G. (GM040505), J.T.L. (GM025232), and D.H.P. (GM035500), and by the Intramural Research Program of the NIH, National Institute of Environmental Health Sciences, to K.A. (Z01 ES101987).

\section{References}

Bentley DL. 2005. Rules of engagement: Co-transcriptional recruitment of pre-mRNA processing factors. Curr Opin Cell Biol 17: 251-256.

Berro R, Pedati C, Kehn-Hall K, Wu W, Klase Z, Even Y, Geneviere AM, Ammosova $T$, Nekhai S, Kashanchi $F$. 2008. CDK13, a new potential human immunodeficiency virus type 1 inhibitory factor regulating viral mRNA splicing. J Virol 82: 7155-7166.

Boehm AK, Saunders A, Werner J, Lis JT. 2003. Transcription factor and polymerase recruitment, modification, and movement on dhsp70 in vivo in the minutes following heat shock. Mol Cell Biol 23: 7628-7637.

Buratowski S. 2009. Progression through the RNA polymerase II CTD cycle. Mol Cell 36: 541-546.

Carty SM, Goldstrohm A, Suñe C, Garcia-Blanco MA, Greenleaf AL. 2000. Protein-interaction modules that organize nuclear function: FF domains of CA150 bind the phospho-CTD of RNA polymerase II. Proc Natl Acad Sci 97: 9015-9020.

Chapman RD, Heidemann M, Albert TK, Mailhammer R, Flatley A, Meisterernst M, Kremmer E, Eick D. 2007.
Transcribing RNA polymerase II is phosphorylated at CTD residue serine-7. Science 318: 1780-1782.

Chen HH, Wang YC, Fann MJ. 2006. Identification and characterization of the CDK12/cyclin L1 complex involved in alternative splicing regulation. Mol Cell Biol 26: 2736-2745.

Chen HH, Wong YH, Geneviere AM, Fann MJ. 2007. CDK13/ CDC2L5 interacts with L-type cyclins and regulates alternative splicing. Biochem Biophys Res Commun 354: 735-740.

Cho EJ, Kobor MS, Kim M, Greenblatt J, Buratowski S. 2001. Opposing effects of Ctk1 kinase and Fcpl phosphatase at Ser 2 of the RNA polymerase II C-terminal domain. Genes \& Dev 15: 3319-3329.

Corden JL. 1990. Tails of RNA polymerase II. Trends Biochem Sci 15: 383-387.

Coudreuse D, van Bakel H, Dewez M, Soutourina J, Parnell T, Vandenhaute J, Cairns B, Werner M, Hermand D. 2010. A gene-specific requirement of RNA polymerase II CTD phosphorylation for sexual differentiation in S. pombe. 20: 10531064.

Egloff S, O'Reilly D, Chapman RD, Taylor A, Tanzhaus K, Pitts L, Eick D, Murphy S. 2007. Serine-7 of the RNA polymerase II CTD is specifically required for snRNA gene expression. Science 318: 1777-1779.

Fu TJ, Peng J, Lee G, Price DH, Flores O. 1999. Cyclin K functions as a CDK9 regulatory subunit and participates in RNA polymerase II transcription. I Biol Chem 274: 3452734530.

Goldstrohm AC, Greenleaf AL, Garcia-blanco MA. 2001. Cotranscriptional splicing of pre-messenger RNAs: Considerations for the mechanism of alternative splicing. Gene 277: 31-47.

Greenleaf AL. 1992. RNA polymerase II subunits, transcription factors, and kinases: Investigations in Drosophila melanogaster and Saccharomyces cerevisiae. In Transcriptional regulation (ed. K Yamamoto, S McKnight), pp. 55-80. Cold Spring Harbor Laboratory Press, Cold Spring Harbor, New York.

Greenleaf AL. 1993. Positive patches and negative noodles: Linking RNA processing to transcription? Trends Biochem Sci 18: 117-119.

Greenleaf AL, Plagens U, Bautz EKF. 1976. Localization of RNA polymerase on Drosophila polytene chromosomes by indirect immunofluorescence. In Molecular mechanisms in the control of gene expression. (ed. DP Nierlich et al.), pp. 249-254. Academic Press, New York.

Grenetier S, Bouchoux C, Goguel V. 2006. CTD kinase I is required for the integrity of the rDNA tandem array. Nucleic Acids Res 34: 4996-5006.

Guo Z, Stiller JW. 2004. Comparative genomics of cyclindependent kinases suggest co-evolution of the RNAP II C-terminal domain and CTD-directed CDKs. BMC 5: 69. doi: 10.1186/1471-2164-5-69.

Hu Y, Raynard S, Sehorn MG, Lu X, Bussen W, Zheng L, Stark JM, Barnes EL, Chi P, Janscak P, et al. 2007. RECQL5/Recq15 helicase regulates homologous recombination and suppresses tumor formation via disruption of Rad51 presynaptic filaments. Genes Dev 21: 3073-3084.

Islam MN, Fox D III, Guo R, Enomoto T, Wang W. 2010. RecQL5 promotes genome stabilization through two parallel mechanisms-interacting with RNA polymerase II and acting as a helicase. Mol Cell Biol 30: 2460-2472.

Jones JC, Phatnani HP, Haystead TA, MacDonald JA, Alam SM, Greenleaf AL. 2004. C-terminal repeat domain kinase I phosphorylates Ser2 and Ser5 of RNA polymerase II C-terminal domain repeats. J Biol Chem 279: 24957-24964.

Kanagaraj R, Huehn D, MacKellar A, Menigatti M, Zheng L, Urban V, Shevelev I, Greenleaf AL, Janscak P. 2010. RECQ5 
helicase associates with the C-terminal repeat domain of RNA polymerase II during productive elongation phase of transcription. Nucl Acids Res doi: 10.1093/nar/gkq697.

Karagiannis J, Balasubramanian MK. 2007. A cyclin-dependent kinase that promotes cytokinesis through modulating phosphorylation of the carboxy terminal domain of the RNA Pol II Rpb1p sub-unit. PLOS ONE 2: e433. doi: 10.1371/journal, pone.0000433.

Kim JB, Sharp PA. 2001. Positive transcription elongation factor B phosphorylates hSPT5 and RNA polymerase II carboxylterminal domain independently of cyclin-dependent kinaseactivating kinase. J Biol Chem 276: 12317-12323.

Kim M, Vasiljeva L, Rando OJ, Zhelkovsky A, Moore C, Buratowski S. 2006. Distinct pathways for snoRNA and mRNA termination. Mol Cell 24: 723-734.

Kizer KO, Phatnani HP, Shibata Y, Hall H, Greenleaf AL, Strahl BD. 2005. A novel domain in Set2 mediates RNA polymerase II interaction and couples histone H3 K36 methylation with transcript elongation. Mol Cell Biol 25: 3305-3316.

Ko TK, Kelly E, Pines J. 2001. CrkRS: A novel conserved Cdc2related protein kinase that colocalises with SC35 speckles. J Cell Sci 114: 2591-2603.

Komarnitsky P, Cho EJ, Buratowski S. 2000. Different phosphorylated forms of RNA polymerase II and associated mRNA processing factors during transcription. Genes Dev 14: 2452-2460.

Lee JM, Greenleaf AL. 1989. A protein kinase that phosphorylates the C-terminal repeat domain of the largest subunit of RNA polymerase II. Proc Natl Acad Sci 86: 3624-3628.

Lee JM, Greenleaf AL. 1991. CTD kinase large subunit is encoded by CTK1, a gene required for normal growth of Saccharomyces cerevisiae. Gene Expr 1: 149-167.

Lis JT, Mason P, Peng J, Price DH, Werner J. 2000. P-TEFb kinase recruitment and function at heat shock loci. Genes Dev 14: 792-803.

Liu J, Kipreos ET. 2000. Evolution of cyclin-dependent kinases (CDKs) and CDK-activating kinases (CAKs): Differential conservation of CAKs in yeast and metazoa. Mol Biol Evol 17: 1061-1074.

Liu Y, Warfield L, Zhang C, Luo J, Allen J, Lang WH, Ranish J, Shokat KM, Hahn S. 2009. Phosphorylation of the transcription elongation factor Spt5 by yeast Burl kinase stimulates recruitment of the PAF complex. Mol Cell Biol 29: 48524863.

Longley MJ, Pierce AJ, Modrich P. 1997. DNA polymerase $\delta$ is required for human mismatch repair in vitro. I Biol Chem 272: 10917-10921.

Morris DP, Lee JM, Sterner DE, Brickey WJ, Greenleaf AL. 1997. Assaying CTD kinases in vitro and phosphorylation-modulated properties of RNA polymerase II in vivo. Methods 12: 264-275.

Munoz MJ, de la Mata M, Kornblihtt AR. 2010. The carboxy terminal domain of RNA polymerase II and alternative splicing. Trends Biochem Sci 35: 497-504.

Oeffinger M, Wei KE, Rogers R, DeGrasse JA, Chait BT, Aitchison JD, Rout MP. 2007. Comprehensive analysis of diverse ribonucleoprotein complexes. Nat Methods 4: 951956.

Patturajan M, Schulte RJ, Sefton BM, Berezney R, Vincent M, Bensaude O, Warren SL, Corden JL. 1998. Growth-related changes in phosphorylation of yeast RNA polymerase II. I Biol Chem 273: 4689-4694.

Patturajan M, Conrad NK, Bregman DB, Corden JL. 1999. Yeast carboxyl-terminal domain kinase I positively and negatively regulates RNA polymerase II carboxyl-terminal domain phosphorylation. I Biol Chem 274: 27823-27828.
Peterlin BM, Price DH. 2006. Controlling the elongation phase of transcription with P-TEFb. Mol Cell 23: 297-305.

Phatnani HP, Greenleaf AL. 2006. Phosphorylation and functions of the RNA polymerase II CTD. Genes Dev 20: 2922-2936.

Phatnani HP, Jones JC, Greenleaf AL. 2004. Expanding the functional repertoire of CTD kinase I and RNA polymerase II: Novel phosphoCTD-associating proteins in the yeast proteome. Biochemistry 43: 15702-15719.

Price DH, Sluder AE, Greenleaf AL. 1987. Fractionation of transcription factors for RNA polymerase II from Drosophila Kc cell nuclear extracts. J Biol Chem 262: 3244-3255.

Qiu H, Hu C, Hinnebusch AG. 2009. Phosphorylation of the Pol II CTD by KIN28 enhances BUR1/BUR2 recruitment and Ser2 CTD phosphorylation near promoters. Mol Cell 33: 752-762.

Rahl PB, Lin CY, Seila AC, Flynn RA, McCuine S, Burge CB, Sharp PA, Young RA. 2010. c-Myc regulates transcriptional pause release. Cell 141: 432-445.

Schroeder SC, Schwer B, Shuman S, Bentley D. 2000. Dynamic association of capping enzymes with transcribing RNA polymerase II. Genes Dev 14: 2435-2440.

Schwartz BE, Werner JK, Lis JT. 2004. Indirect immunofluorescent labeling of Drosophila polytene chromosomes: Visualizing protein interactions with chromatin in vivo. Methods Enzymol 376: 393-404.

Skantar AM, Greenleaf AL. 1995. Identifying a transcription factor interaction site on RNA polymerase II. Gene Expr 5: 49-69.

Steinmetz EJ, Conrad NK, Brow DA, Corden JL. 2001. RNAbinding protein $\mathrm{Nrd} 1$ directs poly(A)-independent 3 '-end formation of RNA polymerase II transcripts. Nature 413:327-331.

Sterner DE, Lee JM, Hardin SE, Greenleaf AL. 1995. The yeast carboxyl-terminal repeat domain kinase CTDK-I is a divergent cyclin-cyclin-dependent kinase complex. Mol Cell Biol 15: 5716-5724.

Viladevall L, St Amour CV, Rosebrock A, Schneider S, Zhang C, Allen JJ, Shokat KM, Schwer B, Leatherwood JK, Fisher RP. 2009. TFIIH and P-TEFb coordinate transcription with capping enzyme recruitment at specific genes in fission yeast. Mol Cell 33: 738-751.

Weeks JR, Hardin SE, Shen J, Lee JM, Greenleaf AL. 1993. Locusspecific variation in phosphorylation state of RNA polymerase II in vivo: Correlations with gene activity and transcript processing. Genes Dev 7: 2329-2344.

Westmoreland TJ, Wickramasekara SM, Guo AY, Selim AL, Winsor TS, Greenleaf AL, Blackwell KL, Olson JA Jr, Marks JR, Bennett CB. 2009. Comparative genome-wide screening identifies a conserved doxorubicin repair network that is diploid specific in Saccharomyces cerevisiae. PLOS ONE 4: e5830. doi: 10.1371/journal.pone.0005830.

Wood A, Shilatifard A. 2006. Bur1/Bur2 and the Ctk complex in yeast: The split personality of mammalian P-TEFb. Cell Cycle 5: 1066-1068.

Yao S, Neiman A, Prelich G. 2000. BUR1 and BUR2 encode a divergent cyclin-dependent kinase-cyclin complex important for transcription in vivo. Mol Cell Biol 20: 7080-7087.

Zehring WA, Lee JM, Weeks JR, Jokerst RS, Greenleaf AL. 1988. The C-terminal repeat domain of RNA polymerase II largest subunit is essential in vivo but is not required for accurate transcription initiation in vitro. Proc Natl Acad Sci 85: 3698-3702.

Zhang J, Corden JL. 1991. Phosphorylation causes a conformational change in the carboxyl-terminal domain of the mouse RNA polymerase II largest subunit. J Biol Chem 266: 2297-2302.

Zhou K, Kuo WH, Fillingham J, Greenblatt JF. 2009. Control of transcriptional elongation and cotranscriptional histone modification by the yeast BUR kinase substrate Spt5. Proc Natl Acad Sci 106: 6956-6961. 


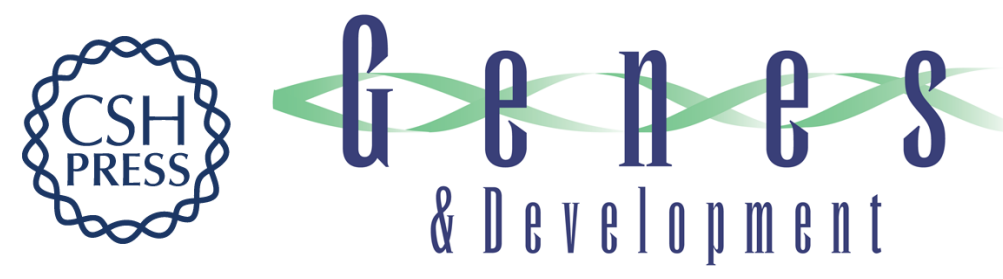

\section{CDK12 is a transcription elongation-associated CTD kinase, the metazoan ortholog of yeast Ctk1}

Bartlomiej Bartkowiak, Pengda Liu, Hemali P. Phatnani, et al.

Genes Dev. 2010, 24:

Access the most recent version at doi:10.1101/gad.1968210

Supplemental http://genesdev.cshlp.org/content/suppl/2010/10/12/24.20.2303.DC1
Material

References This article cites 56 articles, 29 of which can be accessed free at:

http://genesdev.cshlp.org/content/24/20/2303.full.html\#ref-list-1

License

Email Alerting

Receive free email alerts when new articles cite this article - sign up in the box at the top

Service

right corner of the article or click here.

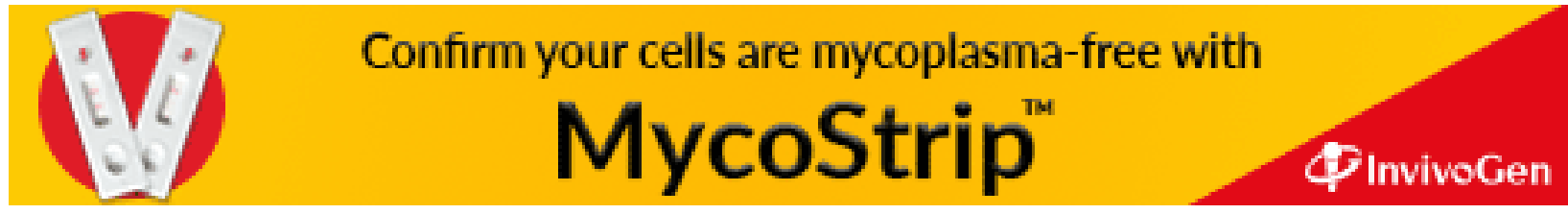

